\title{
Auxiliary Equations Approach for the Stochastic Unsteady Navier-Stokes Equations with Additive Random Noise
}

\author{
Wenju Zhao ${ }^{1,2, *}$ and Max Gunzburger ${ }^{2}$ \\ ${ }^{1}$ Department of Mathematics, Southern University of Science and Technology, \\ Shenzhen, Guangdong 518055, China \\ ${ }^{2}$ Department of Scientific Computing, Florida State University, Tallahassee, \\ FL 32304, USA
}

Received 2 April 2019; Accepted (in revised version) 11 August 2019

\begin{abstract}
This paper presents a Martingale regularization method for the stochastic Navier-Stokes equations with additive noise. The original system is split into two equivalent parts, the linear stochastic Stokes equations with Martingale solution and the stochastic modified Navier-Stokes equations with relatively-higher regularities. Meanwhile, a fractional Laplace operator is introduced to regularize the noise term. The stability and convergence of numerical scheme for the pathwise modified Navier-Stokes equations are proved. The comparisons of non-regularized and regularized noises for the Navier-Stokes system are numerically presented to further demonstrate the efficiency of our numerical scheme.
\end{abstract}

AMS subject classifications: 35R60, 65Mxx, 76Dxx

Key words: Stochastic Navier-Stokes equations, Martingale regularization method, Galerkin finite element method, white noise.

\section{Introduction}

In this paper, a Martingale regularization method is proposed to improve the computational efficiency and accuracy of the stochastic Navier-Stokes equations (SNSEs) with additive noise,

$$
\begin{cases}\mathrm{d} \mathbf{u}-\nu \Delta \mathbf{u} \mathrm{d} t+(\mathbf{u} \cdot \nabla) \mathbf{u} \mathrm{d} t+\nabla p \mathrm{~d} t=\mathbf{f} \mathrm{d} t+\sigma(t) \Delta_{\theta} \mathrm{d} \mathbf{W} & \text { in }(0, T] \times D \times \Omega \\ \nabla \cdot \mathbf{u}=0 & \text { in }[0, T] \times D \times \Omega, \\ \mathbf{u}=\mathbf{0} & \text { on }[0, T] \times \partial D \times \Omega \\ \mathbf{u}=\mathbf{u}_{0} & \text { on } D \times \Omega,\end{cases}
$$

${ }^{*}$ Corresponding author. Email addresses: wz13@my . fsu .edu(W. J. Zhao), mgunzburger@f su . edu (M. Gunzburger) 
where $[0, T]$ denotes a time interval of interest; $D \in \mathbb{R}^{d}, d=2,3$ a space domain with Lipschitz boundary; $\Omega$ a sample space of all the possible outcomes; $\nu$ the viscosity parameter; $\mathbf{u}=\left(u_{1}, \cdots, u_{d}\right):[0, T] \times D \times \Omega \rightarrow \mathbb{R}^{d}$ the velocity field; $p:[0, T] \times D \times \Omega \rightarrow$ $\mathbb{R}$ the corresponding pressure field; $\mathbf{f}:[0, T] \times D \times \Omega \rightarrow \mathbb{R}^{d}$ a random forcing term; $\sigma(t):[0, T] \rightarrow \mathbb{R}$ a scalar function; and $\mathbf{u}_{0}: D \times \Omega \rightarrow \mathbb{R}^{d}$ an initial condition. The notation $\Delta_{\theta}:=(-\Delta)^{-\theta}$ denotes a fractional Laplace operator with $\theta \in \mathbb{R}^{+}[16]$ and $\mathbf{W}=\left(W_{1}, \cdots, W_{d}\right):[0, T] \times D \times \Omega \rightarrow \mathbb{R}^{d}$ denotes an infinite-dimensional Wiener process. Further, assume that $W_{l}, l=1, \cdots, d$ are Wiener processes with bounded, selfadjoint, positive semidefinite covariance operator $Q$ which has eigenvalues $\left\{\gamma_{l}>0\right\}$ and eigenfunctions $\left\{e_{l}(x)\right\}$. Then from [3], the Wiener process $\mathbf{W}$ can be characterized by

$$
\mathbf{W}(t, x, \omega)=\sum_{l=1}^{\infty} \gamma_{l}^{1 / 2} e_{l}(x) \boldsymbol{\beta}_{l}(t, \omega)
$$

where $\boldsymbol{\beta}_{l}(t, \omega)=\left(\beta_{1}^{l}, \cdots, \beta_{d}^{l}\right)$ is a sequence of real-valued independent and identically distributed (i.i.d) standard Brownian motions. In this paper, the trace $\operatorname{tr} Q=\sum_{l=1}^{\infty} \gamma_{l}$ is not required to be finite. The infinite case can be incorporated with some feasible regularization parameter $\theta$ of the fractional Laplace operator.

The crucial part of the Martingale regularization method for (1.1) is having in hand previous knowledge of an auxiliary stochastic process associated with the stochastic Stokes equations

$$
\begin{cases}\mathrm{d} \boldsymbol{\eta}-\nu \Delta \boldsymbol{\eta} \mathrm{d} t+\nabla \zeta \mathrm{d} t=\sigma(t) \Delta_{\theta} \mathrm{d} \mathbf{W} & \text { in }(0, T] \times D \times \Omega, \\ \nabla \cdot \boldsymbol{\eta}=0 & \text { in }[0, T] \times D \times \Omega, \\ \boldsymbol{\eta}=\mathbf{0} & \text { on }[0, T] \times \partial D \times \Omega, \\ \boldsymbol{\eta}=\boldsymbol{\eta}_{0} & \text { on } D \times \Omega,\end{cases}
$$

where $\boldsymbol{\eta}:[0, T] \times D \times \Omega \rightarrow \mathbb{R}^{d}$ is the corresponding auxiliary velocity and $\zeta:[0, T] \times$ $D \times \Omega \rightarrow \mathbb{R}$ is the corresponding pressure. Theoretically, the stochastic Stokes equations (1.3) are somehow relevant to the divergence free projection of one stochastic parabolic equations. Moreover, the solution of Eqs. (1.3) with time-space white noise in two dimension or higher has less regularities, e.g., $\boldsymbol{\eta}(t) \notin L_{\mathbb{P}}^{2}\left(\Omega, \mathbf{H}^{1}(D)\right)$ [3]. The fractional Laplace operator $\Delta_{\theta}$ is given here to regularize the noise term $\mathrm{dW}$ so as to make the pathwise solution of (1.3) smoother and more amenable to computation. Therefore, system (1.1) with a cylindrical Wiener process or a greatly non-smoother Wiener process can be covered. Setting $\mathbf{u}=\boldsymbol{\xi}+\boldsymbol{\eta}, p=\zeta+\pi$, the induced velocity $\boldsymbol{\xi}$ and pressure $\pi$ satisfy the modified Navier-Stokes equations

$$
\begin{cases}\mathrm{d} \boldsymbol{\xi}-\nu \Delta \boldsymbol{\xi} \mathrm{d} t+(\boldsymbol{\xi}+\boldsymbol{\eta}) \cdot \nabla(\boldsymbol{\xi}+\boldsymbol{\eta}) \mathrm{d} t+\nabla \pi \mathrm{d} t=\mathbf{f} \mathrm{d} t & \text { in }(0, T] \times D \times \Omega, \\ \nabla \cdot \boldsymbol{\xi}=0 & \text { in }[0, T] \times D \times \Omega, \\ \boldsymbol{\xi}=\mathbf{0} & \text { on }[0, T] \times \partial D \times \Omega, \\ \boldsymbol{\xi}=\boldsymbol{\xi}_{0} & \text { on } D \times \Omega .\end{cases}
$$


The modified system (1.4) maintains higher regularities than the system (1.1). The Martingale regularization method leads the simulation and analysis of the stochastic Navier-Stokes equations (1.1) to the stochastic Stokes equations (1.3) which are linear and the stochastic modified Navier-Stokes equations (1.4) with smoother noise. For some rigorous theoretical analyses of the stochastic Navier-Stokes equations, readers can refer to $[2,4,5,7,11,13,17]$, etc. Some interesting topics of the stochastic partial differential equations (SPDEs) are further detailed in $[1,6,8,14,15,18]$.

The remainder of this paper is organized as follows. Section 2 specifies the notations and the mathematical formulations of the Navier-Stokes equations throughout the paper. Section 3 presents the properties of the auxiliary equations (stochastic Stokes equations). Section 4 proofs the stability and the convergence of the pathwise solution of the modified Navier-Stokes equations under sufficient regularity assumptions. Section 5 presents the numerical simulations and illustrates the results of numerical experiments for the stochastic Navier-Stokes equations. Section 6 presents some conclusions.

\section{Notations and preliminaries}

Let $L^{2}(D)$ be the space of all real square integrable functions with norm and inner product $\|\cdot\|$ and $(\cdot, \cdot)$, respectively. For $m, k \in \mathbb{N}$, let $H^{m}(D)$ be a classical Sobolev space,

$$
H^{m}(D)=\left\{v \in L^{2}(D) ; \partial^{k} v \in L^{2}(D),|k| \leq m\right\}
$$

equipped with the norm

$$
\|\cdot\|_{m}:=\|\cdot\|_{H^{m}(D)}=\left(\sum_{|k| \leq m}\left\|\partial^{k} v\right\|^{2}\right)^{1 / 2}
$$

and denote by $H_{0}^{m}(D)$ the closure of $C_{c}^{\infty}(D)$ in $H^{m}(D)$. For $s \in \mathbb{R}$, we introduce the fractional Sobolev space [16],

$$
\dot{H}^{s}(D)=\left\{v \in L^{2}(D) ;|v|_{s}^{2}=\sum_{l=1}^{\infty} \lambda_{l}^{s}\left(v, \psi_{l}\right)^{2}<\infty\right\},
$$

where $\left\{\psi_{l}\right\}$ denotes a complete set of orthonormal eigenfunctions in $L^{2}(D)$ and the corresponding increasing eigenvalues $\left\{\lambda_{l}\right\}$ satisfy the Laplace eigenvalue problems

$$
-\Delta \psi_{l}=\lambda_{l} \psi_{l} \quad \text { in } D,\left.\quad \psi_{l}\right|_{\partial D}=0, \quad l=1,2, \cdots .
$$

Further, the power of the negative Laplace operator $-\Delta$ is defined by the combination $\left\{\psi_{l}, \lambda_{l}\right\}$ as $(-\Delta)^{s} v=\sum_{l=1}^{\infty} \lambda_{l}^{s}\left(v, \psi_{l}\right) \psi_{l}$. For additional details about $H_{0}^{m}(D)$ and $\dot{H}^{s}(D)$, 
readers can refer to [16]. To capture the continuity of the random process, for $0<\alpha \leq$ 1 , the Hölder continuous space $C^{k, \alpha}(D)$ is defined as

$$
C^{k, \alpha}(D)=\left\{v \in C^{k}(D) ;\|v\|_{C^{k, \alpha}(D)}<\infty\right\}
$$

with

$$
\|v\|_{C^{k, \alpha}(D)}=\sum_{|\beta| \leq k} \sup _{x \in D}\left|\partial^{\beta} v(x)\right|+\sum_{|\beta|=k}\left[\partial^{\beta} v\right]_{\alpha}
$$

and

$$
[v]_{\alpha}=\sup _{x, y \in D, x \neq y}\left(\frac{|v(x)-v(y)|}{|x-y|^{\alpha}}\right) .
$$

Denote by $\left(\Omega, \mathscr{F}, \mathbb{P},\left\{\mathscr{F}_{t}\right\}_{t \geq 0}\right)$ the complete filtered probability space; $\mathscr{F}$ is the $\sigma$-algebra of events; the filtrations $\left\{\mathscr{F}_{t}\right\}_{t \geq 0}$ is an increasing family of sub- $\sigma$-algebras of $\mathscr{F} ; \mathbb{P}$ : $\mathscr{F} \rightarrow[0,1]$ is a probability measure with $\mathbb{P}(\Omega)=1$. The expectation operator is denoted by $\mathbb{E}[\cdot]:=\int_{\Omega} \cdot \mathrm{d} \mathbb{P}$ on a random variable space. The stochastic Sobolev space $L_{\mathbb{P}}^{2}\left(\Omega, H^{m}(D)\right)$ is given

$$
L_{\mathbb{P}}^{2}\left(\Omega, H^{m}(D)\right)=\left\{v: D \times \Omega \rightarrow \mathbb{R} ; \mathbb{E}\left[\|v\|_{H^{m}(D)}^{2}\right]<+\infty\right\} .
$$

Vector-valued functions and spaces are denoted in bold font, e.g., $\mathbf{v}=\left(v_{1}, \cdots, v_{d}\right)$, $\mathbf{H}^{m}(D)=\left(H^{m}(D)\right)^{d}$. Without ambiguity, no distinction is made in the notations of the norms and inner products, i.e., $\|\cdot\|_{m}$ is used for $\|\cdot\|_{H^{m}(D)}$ or $\|\cdot\|_{\mathbf{H}^{m}(D)}$. Let $\mathbf{X}$ be the velocity space and $Q$ the pressure space as

$$
\mathbf{X}=\left(H_{0}^{1}(D)\right)^{d}, \quad Q:=L_{0}^{2}=\left\{q \in L^{2}(D) ; \int_{D} q \mathrm{~d} D=0\right\} .
$$

The spaces of the divergence free functions are defined as

$$
\begin{aligned}
& \mathbf{V}=\{\mathbf{v} \in \mathbf{X} ;(\nabla \cdot \mathbf{v}, q)=0, \forall q \in Q\} \\
& \mathbf{Z}=\{\mathbf{v} \in \mathbf{X} ; \nabla \cdot \mathbf{v}=0\} .
\end{aligned}
$$

We define

$$
a(\mathbf{u}, \mathbf{v})=\nu(\nabla \mathbf{u}, \nabla \mathbf{v}), \quad b(\mathbf{v}, q)=-(\nabla \cdot \mathbf{v}, q), \quad c(\mathbf{u} ; \mathbf{v}, \mathbf{w})=(\mathbf{u} \cdot \nabla \mathbf{v}, \mathbf{w}) .
$$

The pathwise weak formulations of (1.3) and (1.4) are defined as, for a given sample $\omega \in \Omega$, seek pathwise solutions $\boldsymbol{\eta}(t):=\boldsymbol{\eta}(t, \cdot, \omega) \in \mathbf{X}, \zeta(t):=\zeta(t, \cdot, \omega) \in Q$ satisfying:

$$
\begin{array}{rlrl}
(\boldsymbol{\eta}(t), \mathbf{v}) & =(\boldsymbol{\eta}(0), \mathbf{v})-\int_{0}^{t} a(\boldsymbol{\eta}, \mathbf{v}) \mathrm{d} s-\int_{0}^{t} b(\mathbf{v}, \zeta) \mathrm{d} s & \\
+\int_{0}^{t}\left(\sigma(t) \Delta_{\theta} \mathrm{d} \mathbf{W}, \mathbf{v}\right), & \forall \mathbf{v} \in \mathbf{X}, \\
b(\boldsymbol{\eta}(t), q)=0, & \forall q \in Q,
\end{array}
$$


and seek $\boldsymbol{\xi}(t):=\boldsymbol{\xi}(t, \cdot, \omega) \in \mathbf{X}, \pi(t):=\pi(t, \cdot, \omega) \in Q$ satisfying:

$$
\begin{array}{cl}
(\boldsymbol{\xi}(t), \mathbf{v})=(\boldsymbol{\xi}(0), \mathbf{v})-\int_{0}^{t} a(\boldsymbol{\xi}, \mathbf{v}) \mathrm{d} s-\int_{0}^{t} c(\boldsymbol{\xi}+\boldsymbol{\eta}, \boldsymbol{\xi}+\boldsymbol{\eta}, \mathbf{v}) \mathrm{d} s & \\
-\int_{0}^{t} b(\mathbf{v}, \pi) \mathrm{d} s+\int_{0}^{t}(\mathbf{f}, \mathbf{v}) \mathrm{d} s, & \forall \mathbf{v} \in \mathbf{X}, \\
b(\boldsymbol{\xi}(t), q)=0, & \forall q \in Q .
\end{array}
$$

Let $\left\{T_{h}\right\}_{h>0}$ be a family of regular triangulations of the spatial domain $D$ with $h$ an index of the mesh size of $T_{h}$ (see [17]). We define the conforming finite element spaces $\mathbf{X}_{h} \subset \mathbf{X}$ and $Q_{h} \subset Q$ and the discrete divergence free subspace $\mathbf{V}_{h} \subset \mathbf{X}_{h}$ on $T_{h}$ as

$$
\begin{aligned}
& \mathbf{X}_{h}=\left\{\mathbf{v}_{h} \in\left(C^{0}(D)\right)^{d} ;\left.\mathbf{v}_{h}\right|_{T} \in\left(P^{r+1}(T)\right)^{d}, T \in T_{h}\right\}, \\
& Q_{h}=\left\{q_{h} \in C^{0}(D) \cap Q ;\left.q_{h}\right|_{T} \in P^{r}(T), T \in T_{h}\right\}, \\
& \mathbf{V}_{h}=\left\{\mathbf{v}_{h} \in \mathbf{X}_{h} ;\left(\nabla \cdot \mathbf{v}_{h}, q_{h}\right)=0, \forall q_{h} \in Q_{h}\right\},
\end{aligned}
$$

where $r \in \mathbb{N}^{+}$. For $\mathbf{v} \in \mathbf{H}_{0}^{1}(D), \mathbf{w} \in \mathbf{H}_{0}^{1}(D)$ and $\mathbf{u}$ weakly divergence free, we have

$$
\begin{aligned}
& c(\mathbf{u} ; \mathbf{v}, \mathbf{w})=-c(\mathbf{u} ; \mathbf{w}, \mathbf{v}) \\
& \widetilde{c}(\mathbf{u} ; \mathbf{v}, \mathbf{w})=\frac{1}{2}(c(\mathbf{u} ; \mathbf{v}, \mathbf{w})-c(\mathbf{u} ; \mathbf{w}, \mathbf{v})) \text { skew-symmetric form. }
\end{aligned}
$$

Then, for $\mathbf{u}, \mathbf{v}, \mathbf{w} \in \mathbf{H}^{1}(D)$ there exists a constant $C>0$ such that

$$
|\widetilde{c}(\mathbf{u} ; \mathbf{v}, \mathbf{w})| \leq C\|\nabla \mathbf{u}\|_{\mathbf{L}^{2}(D)}\|\nabla \mathbf{v}\|_{\mathbf{L}^{2}(D)}\|\nabla \mathbf{w}\|_{\mathbf{L}^{2}(D)} .
$$

The same estimate holds for $c(\mathbf{u}, \mathbf{v}, \mathbf{w})$.

The semi-discrete finite element approximations of (2.1) and (2.2) can be defined. Then for the stochastic Stokes equations, we seek $\boldsymbol{\eta}_{h}(t):=\boldsymbol{\eta}_{h}(t, \cdot, \omega) \in \mathbf{X}_{h}$ and $\zeta_{h}(t):=$ $\zeta_{h}(t, \cdot, \omega) \in Q_{h}$ such that

$$
\begin{array}{cl}
\left(\boldsymbol{\eta}_{h}(t), \mathbf{v}_{h}\right)=\left(\boldsymbol{\eta}_{h}(0), \mathbf{v}_{h}\right)-\int_{0}^{t} a\left(\boldsymbol{\eta}_{h}, \mathbf{v}_{h}\right) \mathrm{d} s-\int_{0}^{t} b\left(\mathbf{v}_{h}, \zeta_{h}\right) \mathrm{d} s & \\
+\int_{0}^{t}\left(\sigma(t) \Delta_{\theta} \mathrm{d} \mathbf{W}, \mathbf{v}_{h}\right), & \forall \mathbf{v}_{h} \in \mathbf{X}_{h}, \\
b\left(\boldsymbol{\eta}_{h}(t), q_{h}\right)=0, & \forall q_{h} \in Q_{h},
\end{array}
$$

and for the stochastic modified Navier-Stokes equations, seek $\boldsymbol{\xi}_{h}(t)=\boldsymbol{\xi}(t, \cdot, \omega) \in \mathbf{X}_{h}$ and $\pi_{h}(t)=\pi_{h}(t, \cdot, \omega) \in Q_{h}$, such that

$$
\begin{array}{cc}
\left(\boldsymbol{\xi}_{h}(t), \mathbf{v}_{h}\right)=\left(\boldsymbol{\xi}_{h}(0), \mathbf{v}_{h}\right)-\int_{0}^{t} a\left(\boldsymbol{\xi}_{h}, \mathbf{v}_{h}\right) \mathrm{d} s-\int_{0}^{t} \widetilde{c}\left(\boldsymbol{\xi}_{h}+\boldsymbol{\eta}_{h} ; \boldsymbol{\xi}_{h}+\boldsymbol{\eta}_{h}, \mathbf{v}_{h}\right) \mathrm{d} s \\
-\int_{0}^{t} b\left(\mathbf{v}_{h}, \pi_{h}\right) \mathrm{d} s+\int_{0}^{t}\left(\mathbf{f}, \mathbf{v}_{h}\right) \mathrm{d} s, & \forall \mathbf{v}_{h} \in \mathbf{X}_{h}, \\
b\left(\boldsymbol{\xi}_{h}(t), q_{h}\right)=0, & \forall q_{h} \in Q_{h} .
\end{array}
$$


Let $I_{h}: \mathbf{H}^{k}(D) \cap \dot{\mathbf{H}}^{\beta+1}(D) \rightarrow \mathbf{V}_{h}, J_{h}: L_{0}^{2} \cap \dot{H}^{\beta}(D) \rightarrow Q_{h}$ be two interpolation operators (see [16]). For $\forall \mathbf{v} \in \mathbf{X}, \forall q \in Q$, assume that the interpolation operators $I_{h}: \mathbf{X} \cap \dot{\mathbf{H}}^{\beta+1}(D) \rightarrow \mathbf{V}_{h}$ and $J_{h}: Q \cap \dot{\mathbf{H}}^{\beta}(D) \rightarrow Q_{h}$ satisfy the estimates

$$
\begin{aligned}
& \left\|I_{h} \mathbf{v}-\mathbf{v}\right\|_{\mathbf{L}^{2}(D)} \leq C h^{\beta+1}\|\mathbf{v}\|_{\dot{H}^{\beta+1}(D)}, \\
& \left\|I_{h} \mathbf{v}-\mathbf{v}\right\|_{\mathbf{H}^{1}(D)}+\left\|J_{h} q-q\right\|_{L^{2}(D)} \leq C h^{\beta}\left(\|\mathbf{v}\|_{\dot{H}^{\beta+1}(D)}+\|q\|_{\dot{H}^{\beta}(D)}\right),
\end{aligned}
$$

and for $\forall \mathbf{v}_{h} \in \mathbf{X}_{h}, \forall q_{h} \in Q_{h}$, the usual discrete inf-sup condition is true,

$$
\inf _{q_{h} \in Q_{h}} \sup _{\mathbf{v}_{h} \in \mathbf{X}_{h}} \frac{b\left(\mathbf{v}_{h}, q_{h}\right)}{\left\|\mathbf{v}_{h}\right\|_{\mathbf{X}}\left\|q_{h}\right\|_{Q}}>0 .
$$

Lemma 2.1 (Inverse inequality). Let $\left\{T_{h}\right\}_{h>0}$ be a quasi-uniform family of triangulations of $D$. There exists constant $C>0$ such that for $\mathbf{v}_{h} \in \mathbf{X}_{h}$,

$$
\left\|\nabla \mathbf{v}_{h}\right\|_{\mathbf{L}^{2}(D)}^{2} \leq C h^{-2}\left\|\mathbf{v}_{h}\right\|_{\mathbf{L}^{2}(D)}^{2} .
$$

Lemma 2.2 (Poincaré inequality). Let $D$ be a bounded domain, then there exists a positive constant $C$ such that, for $\mathbf{v} \in \mathbf{H}_{0}^{k}(D), k \geq 1$,

$$
\|\mathbf{v}\|_{\mathbf{L}^{2}(D)} \leq C\|\nabla \mathbf{v}\|_{\mathbf{L}_{2}(D)} \text {. }
$$

Lemma 2.3 ([12]). Let $\mathscr{P}$ be a divergence free projection operator, then there is a constant $C>0$ so that for all $\mathbf{v} \in \mathbf{H}^{k}(D)$,

$$
\|\mathscr{P} \mathbf{v}\|_{\mathbf{H}^{k}(D)} \leq C\|\mathbf{v}\|_{\mathbf{H}^{k}(D)} .
$$

Lemma 2.4 (Discrete Gronwall lemma, no $\Delta t$-restriction [9]). Let $C \geq 0$ and $a_{l}, b_{l}, d_{l}$ $\geq 0$ for integer $l \in \mathbb{N}$, be nonnegative numbers such that

$$
a_{N}+\Delta t \sum_{l=0}^{N} b_{l} \leq \Delta t \sum_{l=0}^{N-1} a_{l} d_{l}+C, \quad \forall N \in \mathbb{N}^{+} .
$$

Then

$$
a_{N}+\Delta t \sum_{l=0}^{N} b_{l} \leq C \exp \left(\Delta t \sum_{l=0}^{N-1} d_{l}\right)
$$

\section{The properties of the stochastic Stokes equations}

In this part, we consider the properties of the stochastic Stokes equations (1.3). Applying the divergence free projection operator $\mathscr{P}: L^{2}\left(\Omega, \mathbf{H}^{1}(D)\right) \rightarrow L^{2}(\Omega, \mathbf{Z})$ to the Eqs. (1.3), we have the following equivalent system

$$
\left\{\begin{array}{l}
\mathrm{d} \boldsymbol{\eta}-\nu \mathscr{P} \Delta \boldsymbol{\eta} \mathrm{d} t=\sigma(t) \mathscr{P} \Delta_{\theta} \mathrm{d} \mathbf{W} \\
\boldsymbol{\eta}(0)=\boldsymbol{\eta}_{0}
\end{array}\right.
$$


In the space-periodic case, $\mathscr{P} \Delta \boldsymbol{\eta}=\Delta \boldsymbol{\eta}$. Let $S(t)=e^{t \nu \mathscr{P} \Delta}$ be an analytic semigroup. (3.1) admits an explicit mild solution [3]

$$
\boldsymbol{\eta}(t)=S(t) \boldsymbol{\eta}_{0}+\int_{0}^{t} S(t-s) \sigma(t) \mathscr{P} \Delta_{\theta} \mathrm{d} \mathbf{W}(s), \quad \boldsymbol{\eta}(t) \in L_{\mathbb{P}}^{2}(\Omega, \mathbf{Z}) .
$$

From Lemma 2.3, for $\boldsymbol{\eta} \in L_{\mathbb{P}}^{2}(\Omega, \mathbf{Z})$ in (3.1), then there exists a $\mathbf{v} \in L_{\mathbb{P}}^{2}(\Omega, \mathbf{X})$ in (3.3) such that

$$
\|\boldsymbol{\eta}\|_{L_{\mathbb{P}}^{2}\left(\Omega, \mathbf{L}^{2}(D)\right)} \leq\|\mathbf{v}\|_{L_{\mathbb{P}}^{2}\left(\Omega, \mathbf{L}^{2}(D)\right)}
$$

\subsection{Explicit solution of the stochastic parabolic equation}

Based on Lemma 2.3, let us consider the following stochastic parabolic equation

$$
\left\{\begin{array}{l}
\mathrm{d} \mathbf{v}-\nu \Delta \mathbf{v} \mathrm{d} t=\sigma(t) \Delta_{\theta} \mathrm{d} \mathbf{W} \\
\mathbf{v}(0)=\mathbf{0}
\end{array}\right.
$$

where the viscosity $\nu$ and the random term are the same as that in (1.3). To avoid certain technicalities, we assume that the covariance operator $Q$ in (1.2) and negative Laplace operator $-\Delta$ admit the same orthonormal eigenfunctions $\left\{\psi_{l}\right\}$. The case of general eigenfunctions can be identically determined by $\left\{\psi_{l}\right\}$. Analogous to the polynomial chaos expansion [10], let

$$
\mathbf{v}(t, x, \omega)=\sum_{l=1}^{\infty} \psi_{l}(x) \mathbf{v}_{l}(t, \omega)
$$

and substitute it into (3.3). It follows that the coefficient functions $\mathbf{v}_{l}(t):=\mathbf{v}_{l}(t, \omega)$, $l=1,2, \cdots$, are Ornstein-Uhlenbeck processes:

$$
\left\{\begin{array}{l}
\mathrm{d} \mathbf{v}_{l}(t)+\nu \lambda_{l} \mathbf{v}_{l} \mathrm{~d} t=\sigma(t) \sqrt{\gamma_{l}} \lambda_{l}^{-\theta} \mathrm{d} \boldsymbol{\beta}_{l} \\
\mathbf{v}_{l}(0)=\mathbf{0}
\end{array}\right.
$$

From [10], (3.4) admits an explicit solution

$$
\mathbf{v}_{l}(t)=\mathbf{v}_{l}(0) e^{-\nu \lambda_{l} t}+\sqrt{\gamma_{l}} \lambda_{l}^{-\theta} \int_{0}^{t} \sigma(\tau) e^{-\nu \lambda_{l}(t-\tau)} \mathrm{d} \boldsymbol{\beta}_{l}(\tau)
$$

and for each component of $\mathbf{v}_{l}=\left(v_{1}^{l}, \cdots, v_{d}^{l}\right)$, with $C_{\sigma}=\max _{t \in[0, T]}|\sigma(t)|$, the variance functions are bounded as

$$
\operatorname{Var}\left(v_{i}^{l}(t)\right) \leq \frac{C_{\sigma}^{2} \gamma_{l}}{2 \nu \lambda_{l}^{1+2 \theta}}\left(1-e^{-2 \nu \lambda_{l} t}\right), \quad i=1, \cdots, d .
$$

In sum, (3.3) admits an explicit solution as

$$
\mathbf{v}(t, x, \omega)=\sum_{l=1}^{\infty} \psi_{l}(x) \sqrt{\gamma_{l}} \lambda_{l}^{-\theta} \int_{0}^{t} \sigma(\tau) e^{-\nu \lambda_{l}(t-\tau)} \mathrm{d} \boldsymbol{\beta}_{l}(\tau) .
$$

Let us further consider the properties of the explicit solution of (3.7). 
Lemma 3.1. Assume that the orthonormal eigenfunctions $\left\{\psi_{l}\right\} \in C^{1}(D)$ and

$$
\left|\psi_{l}(x)\right| \leq C, \quad\left|\nabla \psi_{l}(x)\right| \leq C \sqrt{\lambda_{l}} \quad \text { and } \quad \sigma(t)<C_{\sigma}, \quad t \in[0, T]
$$

If $\sum_{l=1}^{\infty} \frac{\gamma_{l}}{\lambda_{l}^{1+2 \theta}}$ and $\sum_{l=1}^{\infty} \frac{\gamma_{l}}{\lambda_{l}^{1+2 \theta-\vartheta}}$ are finite for some $\vartheta \in(0,1)$, then

$$
\mathbb{E}\left[|\mathbf{v}(t, x)-\mathbf{v}(s, y)|^{2}\right] \leq C\left\{|t-s|^{2}+|x-y|^{2}\right\}^{\vartheta / 2}
$$

and $\mathbf{v}(t)$ has a version with $\alpha$-Hölder continuous path for $\alpha \in\left(0, \frac{\vartheta}{2}\right)$.

Proof. We follow the proof procedure in [3] and the constant $C>0$ may be different. The series (3.7) converges in $L_{\mathbb{P}}^{2}\left(\Omega, \mathbf{L}^{2}(D)\right)$ for all $t \geq 0$ and $x \in D$,

$$
\begin{aligned}
\|\mathbf{v}\|_{L_{\mathbb{P}}^{2}\left(\Omega, \mathbf{L}^{2}(D)\right)}^{2} & \leq \sum_{l=1}^{\infty} \frac{d C^{2} C_{\sigma}^{2} \gamma_{l}}{2 \nu \lambda_{l}^{1+2 \theta}}\left(1-e^{-2 \nu \lambda_{l} t}\right) \\
& \leq \frac{d C^{2} C_{\sigma}^{2}}{2 \nu} \sum_{l=1}^{\infty} \frac{\gamma_{l}}{\lambda_{l}^{1+2 \theta}}<+\infty
\end{aligned}
$$

With the interpolation arguments, for all $\vartheta \in[0,1]$, we have

$$
\begin{array}{ll}
\left|\psi_{l}(x)-\psi_{l}(y)\right| \leq C 2^{1-\vartheta} \lambda_{l}^{\vartheta / 2}|x-y|^{\vartheta}, & \forall x, y \in D, \\
\left|e^{-t}-e^{-s}\right| \leq C_{\vartheta}|t-s|^{\vartheta}, \quad C_{\vartheta}>0, & \forall t \geq 0, \quad s \geq 0 .
\end{array}
$$

For different $x, y \in D$, with Itô isometry property, we have

$$
\begin{aligned}
& \mathbb{E}\left[|\mathbf{v}(t, x)-\mathbf{v}(t, y)|^{2}\right] \\
= & \sum_{l=1}^{\infty} d\left|\psi_{l}(x)-\psi_{l}(y)\right|^{2} \gamma_{l} \lambda_{l}^{-2 \theta} \int_{0}^{t} \sigma^{2}(\tau) e^{-2 \nu \lambda_{l}(t-\tau)} \mathrm{d} \tau \\
\leq & \sum_{l=1}^{\infty} d C_{\sigma}^{2} C^{2} 2^{2-\vartheta} \lambda_{l}^{\vartheta / 2}|x-y|^{\vartheta} \gamma_{l} \lambda_{l}^{-2 \theta} \int_{0}^{t} e^{-2 \nu \lambda_{l}(t-\tau)} \mathrm{d} \tau \\
\leq & \frac{C}{2 \nu} \sum_{l=1}^{\infty} \frac{\gamma_{l}}{\lambda_{l}^{1+2 \theta-\vartheta / 2}}|x-y|^{\vartheta}
\end{aligned}
$$

with

$$
|\mathbf{v}(t, x)-\mathbf{v}(t, y)|^{2}:=\sum_{l=1}^{d}\left|v_{l}(t, x)-v_{l}(t, y)\right|^{2}
$$


Then for $0 \leq s \leq t \leq T$, we have

$$
\begin{aligned}
& \mathbb{E}\left[|\mathbf{v}(t, x)-\mathbf{v}(s, x)|^{2}\right] \\
= & \sum_{l=1}^{\infty} d\left|\psi_{l}(x)\right|^{2} \gamma_{l} \lambda_{l}^{-2 \theta} \int_{s}^{t} \sigma^{2}(\tau) e^{-2 \nu \lambda_{l}(t-\tau)} \mathrm{d} \tau \\
& \quad+\sum_{l=1}^{\infty} d\left|\psi_{l}(x)\right|^{2} \gamma_{l} \lambda_{l}^{-2 \theta} \int_{0}^{s}\left|\sigma(\tau) e^{-\nu \lambda_{l}(t-\tau)}-\sigma(\tau) e^{-\nu \lambda_{l}(s-\tau)}\right|^{2} \mathrm{~d} \tau \\
= & : d I_{1}(t)+d I_{2}(t, s) .
\end{aligned}
$$

For each term, we have

$$
\begin{aligned}
I_{1}(t) & \leq C_{\sigma}^{2} \sum_{l=1}^{\infty}\left|\psi_{l}(x)\right|^{2} \gamma_{l} \lambda_{l}^{-2 \theta} \int_{s}^{t} e^{-2 \nu \lambda_{l}(t-\tau)} \mathrm{d} \tau \\
& =C_{\sigma}^{2} C^{2} \sum_{l=1}^{\infty} \frac{\gamma_{l}}{2 \nu \lambda_{l}^{1+2 \theta}}\left(1-e^{-2 \nu \lambda_{l}(t-s)}\right) \\
& \leq \frac{C_{\sigma}^{2} C^{2} C_{\vartheta}}{(2 \nu)^{1-\vartheta}} \sum_{l=1}^{\infty} \frac{\gamma_{l}}{\lambda_{l}^{1+2 \theta-\vartheta}}|t-s|^{\vartheta} .
\end{aligned}
$$

Moreover,

$$
\begin{aligned}
I_{2}(t, s) & \leq C_{\sigma}^{2} \sum_{l=1}^{\infty}\left|\psi_{l}(x)\right|^{2} \gamma_{l} \lambda_{l}^{-2 \theta} \int_{0}^{s}\left|e^{-\nu \lambda_{l}(t-\tau)}-e^{-\nu \lambda_{l}(s-\tau)}\right|^{2} \mathrm{~d} \tau \\
& \leq \sum_{l=1}^{\infty} 2 C^{2} C_{\sigma}^{2} \frac{\gamma_{l}}{\nu \lambda_{l}^{1+2 \theta}}\left(1-e^{-\nu \lambda_{l}(t-s)}\right) \leq C \sum_{l=1}^{\infty} \frac{\gamma_{l}}{\lambda_{l}^{1+2 \theta-\vartheta}}|t-s|^{\vartheta} .
\end{aligned}
$$

Combing the above inequalities and applying Kolmogorov's test [3], we have that the random process $\mathbf{v}$ of (3.3) has a version with $\alpha$-Hol̈der continuous path with respect to $t \geq 0, x \in D$ and any $\alpha \in\left(0, \frac{\vartheta}{2}\right)$.

Corollary 3.1. When the regularization parameter $\theta=0$, the stochastic parabolic equation (3.3) with a time-space white noise (cylindrical Wiener process) with $\gamma_{l}=C \neq 0$, $l=1, \cdots$, has a solution $\mathbf{v}(t) \notin L_{\mathbb{P}}^{2}\left(\Omega, \mathbf{L}^{2}(D)\right)$ for $d \geq 2$. Moreover, with some regularization parameter $\theta>0$, (3.3) can be guaranteed to be sufficiently smooth.

\section{Numerical analyses for the pathwise modified Navier-Stokes equations}

Under sufficient assumptions in Lemma 3.1, the stochastic Stokes equations (1.3) have a $\alpha$-hölder-continuous solution. Further, the modified Navier-Stokes equations (1.4) have somehow higher regularities. In this section, we investigate the properties 
of the pathwise modified Navier-Stokes equations under weak regularity assumptions which are consistent with Lemma 3.1. We assume the following regularities

$$
\begin{aligned}
& \boldsymbol{\eta} \in C^{0, \alpha}\left([0, T] ; \dot{\mathbf{H}}^{\beta}(D) \cap \mathbf{H}_{0}^{1}(D)\right), \quad \zeta \in C^{0, \alpha}\left([0, T] ; L^{2}(D)\right), \\
& \boldsymbol{\xi} \in C^{1, \alpha}\left([0, T] ; \dot{\mathbf{H}}^{\beta+1}(D) \cap \mathbf{H}^{2}(D) \cap \mathbf{H}_{0}^{1}(D)\right), \quad \pi \in C^{1, \alpha}\left([0, T] ; L^{2}(D)\right), \\
& \mathbf{f} \in L^{2}\left([0, T] ; \mathbf{L}^{2}(D)\right), \quad 0<\beta<1 .
\end{aligned}
$$

In the following, we assume that the solution of the stochastic Stokes equations is exactly true and that $\mathbf{u}_{h}=\boldsymbol{\xi}_{h}+\boldsymbol{\eta}$, where $\boldsymbol{\eta}$ is a solution of the stochastic Stokes equations (1.3). Let $t_{n}=n \Delta t, n=0,1, \cdots, N-1$ and $t_{N}=N \Delta t=T$ and $\boldsymbol{\xi}^{n}=\boldsymbol{\xi}\left(t_{n}\right)$, $\boldsymbol{\xi}^{n+\frac{1}{2}}=\frac{\boldsymbol{\xi}\left(t_{n}\right)+\boldsymbol{\xi}\left(t_{n+1}\right)}{2}, \boldsymbol{\xi}_{h}^{n+\frac{1}{2}}=\frac{\boldsymbol{\xi}_{h}^{n}+\boldsymbol{\xi}_{h}^{n+1}}{2}$, analogous to $\boldsymbol{\eta}$, f, respectively. We have a full discrete scheme of (2.4) as follows: for a given $\boldsymbol{\xi}_{h}^{n} \in \mathbf{X}_{h}$, seek $\boldsymbol{\xi}_{h}^{n+1} \in \mathbf{X}_{h}, \pi_{h}^{n+1} \in Q_{h}$ such that

$$
\begin{array}{ll}
\left(\frac{\boldsymbol{\xi}_{h}^{n+1}-\boldsymbol{\xi}_{h}^{n}}{\Delta t}, \mathbf{v}_{h}\right)+a\left(\boldsymbol{\xi}_{h}^{n+\frac{1}{2}}, \mathbf{v}_{h}\right)+\widetilde{c}\left(\mathbf{u}_{h}^{n} ; \mathbf{u}_{h}^{n+\frac{1}{2}}, \mathbf{v}_{h}\right)+b\left(\mathbf{v}_{h}, \pi_{h}^{n+1}\right) & \\
\quad=\left(\mathbf{f}\left(t_{n+\frac{1}{2}}\right), \mathbf{v}_{h}\right), & \mathbf{v}_{h} \in \mathbf{X}_{h}, \\
b\left(\boldsymbol{\xi}_{h}^{n+1}, q_{h}\right)=0, & q_{h} \in Q_{h} .
\end{array}
$$

Theorem 4.1 (Stability). Assume that $\boldsymbol{\xi}_{h}^{0} \in \mathbf{V}_{h}$ and $\|\nabla \boldsymbol{\eta}(t)\|<C_{\boldsymbol{\eta}}<+\infty, t \in[0, T]$, then there exists a constant $C$ such that

$$
\begin{aligned}
& \left\|\boldsymbol{\xi}_{h}^{N}\right\|^{2}+\nu \Delta t \sum_{n=0}^{N-1}\left\|\nabla \boldsymbol{\xi}_{h}^{n+\frac{1}{2}}\right\|^{2} \\
\leq & C \exp \left(\frac{C T}{\nu h^{2}}\right)\left(\frac{T}{\nu}+\frac{\Delta t}{\nu} \sum_{n=0}^{N-1}\left\|\mathbf{f}\left(t_{n+\frac{1}{2}}\right)\right\|^{2}+\left\|\boldsymbol{\xi}_{h}^{0}\right\|^{2}\right) .
\end{aligned}
$$

Proof. Set $\left(\mathbf{v}_{h}, q_{h}\right)=2 \Delta t\left(\boldsymbol{\xi}_{h}^{n+\frac{1}{2}}, \pi_{h}^{n+1}\right) \in \mathbf{V}_{h} \times Q_{h}$ in (4.1). The skew-symmetric form of the convective term vanishes

$$
\widetilde{c}\left(\mathbf{u}_{h}^{n} ; \boldsymbol{\xi}_{h}^{n+\frac{1}{2}}, \boldsymbol{\xi}_{h}^{n+\frac{1}{2}}\right)=0 .
$$

Since $b\left(\boldsymbol{\xi}_{h}^{n}, q_{h}\right)=0, q_{h} \in Q_{h}$, it follows that $b\left(\mathbf{v}_{h}, \pi_{h}^{n+1}\right)=0$. Then, we have the following estimates

$$
\begin{aligned}
& \left\|\boldsymbol{\xi}_{h}^{n+1}\right\|^{2}-\left\|\boldsymbol{\xi}_{h}^{n}\right\|^{2}+2 \nu \Delta t\left\|\nabla \boldsymbol{\xi}_{h}^{n+\frac{1}{2}}\right\|^{2} \\
= & -2 \Delta t \widetilde{c}\left(\mathbf{u}_{h}^{n} ; \mathbf{u}_{h}^{n+\frac{1}{2}}, \boldsymbol{\xi}_{h}^{n+\frac{1}{2}}\right)+2 \Delta t\left(\mathbf{f}\left(t_{n+\frac{1}{2}}\right), \boldsymbol{\xi}_{h}^{n+\frac{1}{2}}\right) \\
= & -2 \Delta t \widetilde{c}\left(\mathbf{u}_{h}^{n} ; \boldsymbol{\eta}^{n+\frac{1}{2}}, \boldsymbol{\xi}_{h}^{n+\frac{1}{2}}\right)+2 \Delta t\left(\mathbf{f}\left(t_{n+\frac{1}{2}}\right), \boldsymbol{\xi}_{h}^{n+\frac{1}{2}}\right) \\
= & -2 \Delta t \widetilde{c}\left(\boldsymbol{\xi}_{h}^{n} ; \boldsymbol{\eta}^{n+\frac{1}{2}}, \boldsymbol{\xi}_{h}^{n+\frac{1}{2}}\right)-2 \Delta t \widetilde{c}\left(\boldsymbol{\eta}^{n} ; \boldsymbol{\eta}^{n+\frac{1}{2}}, \boldsymbol{\xi}_{h}^{n+\frac{1}{2}}\right)+2 \Delta t\left(\mathbf{f}\left(t_{n+\frac{1}{2}}\right), \boldsymbol{\xi}_{h}^{n+\frac{1}{2}}\right) \\
\leq & 2 \Delta t\left|\widetilde{c}\left(\boldsymbol{\xi}_{h}^{n} ; \boldsymbol{\eta}^{n+\frac{1}{2}}, \boldsymbol{\xi}_{h}^{n+\frac{1}{2}}\right)\right|+2 \Delta t\left|\widetilde{c}\left(\boldsymbol{\eta}^{n} ; \boldsymbol{\eta}^{n+\frac{1}{2}}, \boldsymbol{\xi}_{h}^{n+\frac{1}{2}}\right)\right|+2 \Delta t\left|\left(\mathbf{f}\left(t_{n+\frac{1}{2}}\right), \boldsymbol{\xi}_{h}^{n+\frac{1}{2}}\right)\right| .
\end{aligned}
$$


For the convection terms, with Lemma 2.1, we have the following estimates,

$$
\begin{aligned}
\widetilde{c}\left(\boldsymbol{\xi}_{h}^{n} ; \boldsymbol{\eta}^{n+\frac{1}{2}}, \boldsymbol{\xi}_{h}^{n+\frac{1}{2}}\right) & \leq C\left\|\nabla \boldsymbol{\xi}_{h}^{n}\right\|\left\|\nabla \boldsymbol{\eta}^{n+\frac{1}{2}}\right\|\left\|\nabla \boldsymbol{\xi}_{h}^{n+\frac{1}{2}}\right\| \\
& \leq \frac{\nu}{6}\left\|\nabla \boldsymbol{\xi}_{h}^{n+\frac{1}{2}}\right\|^{2}+\frac{C}{\nu}\left\|\nabla \boldsymbol{\xi}_{h}^{n}\right\|^{2}\left\|\nabla \boldsymbol{\eta}^{n+\frac{1}{2}}\right\|^{2} \\
& \leq \frac{\nu}{6}\left\|\nabla \boldsymbol{\xi}_{h}^{n+\frac{1}{2}}\right\|^{2}+\frac{C}{\nu h^{2}}\left\|\boldsymbol{\xi}_{h}^{n}\right\|^{2}\left\|\nabla \boldsymbol{\eta}^{n+\frac{1}{2}}\right\|^{2}, \\
\widetilde{c}\left(\boldsymbol{\eta}^{n} ; \boldsymbol{\eta}^{n+\frac{1}{2}}, \boldsymbol{\xi}_{h}^{n+\frac{1}{2}}\right) & \leq\left\|\nabla \boldsymbol{\eta}^{n}\right\|\left\|\nabla \boldsymbol{\eta}^{n+\frac{1}{2}}\right\|\left\|\nabla \boldsymbol{\xi}_{h}^{n+\frac{1}{2}}\right\| \\
& \leq \frac{\nu}{6}\left\|\nabla \boldsymbol{\xi}_{h}^{n+\frac{1}{2}}\right\|^{2}+\frac{C}{\nu}\left\|\nabla \boldsymbol{\eta}^{n}\right\|^{2}\left\|\nabla \boldsymbol{\eta}^{n+\frac{1}{2}}\right\|^{2} .
\end{aligned}
$$

For the forcing term, we have

$$
\left(\mathbf{f}\left(t_{n+\frac{1}{2}}\right), \boldsymbol{\xi}_{h}^{n+\frac{1}{2}}\right) \leq\left\|\mathbf{f}\left(t_{n+\frac{1}{2}}\right)\right\|\left\|\boldsymbol{\xi}_{h}^{n+\frac{1}{2}}\right\| \leq \frac{\nu}{6}\left\|\nabla \boldsymbol{\xi}_{h}^{n+\frac{1}{2}}\right\|^{2}+\frac{C}{\nu}\left\|\mathbf{f}\left(t_{n+\frac{1}{2}}\right)\right\|^{2}
$$

Combing the above inequalities together gives

$$
\begin{aligned}
& \left\|\boldsymbol{\xi}_{h}^{n+1}\right\|^{2}-\left\|\boldsymbol{\xi}_{h}^{n}\right\|^{2}+\nu \Delta t\left\|\nabla \boldsymbol{\xi}_{h}^{n+\frac{1}{2}}\right\|^{2} \\
\leq & \frac{2 C \Delta t}{\nu h^{2}}\left\|\boldsymbol{\xi}_{h}^{n}\right\|^{2}\left\|\nabla \boldsymbol{\eta}^{n+\frac{1}{2}}\right\|^{2}+\frac{2 C \Delta t}{\nu}\left\|\nabla \boldsymbol{\eta}^{n}\right\|^{2}\left\|\nabla \boldsymbol{\eta}^{n+\frac{1}{2}}\right\|^{2}+\frac{2 C \Delta t}{\nu}\left\|\mathbf{f}\left(t_{n+\frac{1}{2}}\right)\right\|^{2} .
\end{aligned}
$$

Summing above inequalities from $n=0,1, \cdots, N-1$, obtains

$$
\begin{aligned}
& \left\|\boldsymbol{\xi}_{h}^{N}\right\|^{2}-\left\|\boldsymbol{\xi}_{h}^{0}\right\|^{2}+\nu \Delta t \sum_{n=0}^{N-1}\left\|\nabla \boldsymbol{\xi}_{h}^{n+\frac{1}{2}}\right\|^{2} \\
\leq & \frac{2 C \Delta t}{\nu h^{2}} \sum_{n=0}^{N-1}\left\|\boldsymbol{\xi}_{h}^{n}\right\|^{2}\left\|\nabla \boldsymbol{\eta}^{n+\frac{1}{2}}\right\|^{2}+\frac{2 C \Delta t}{\nu} \sum_{n=0}^{N-1}\left\|\nabla \boldsymbol{\eta}^{n}\right\|^{2}\left\|\nabla \boldsymbol{\eta}^{n+\frac{1}{2}}\right\|^{2}+\frac{2 C \Delta t}{\nu} \sum_{n=0}^{N-1}\left\|\mathbf{f}\left(t_{n+\frac{1}{2}}\right)\right\|^{2} .
\end{aligned}
$$

With the Lemma 2.4, we have

$$
\begin{gathered}
\left\|\boldsymbol{\xi}_{h}^{N}\right\|^{2}+\nu \Delta t \sum_{n=0}^{N-1}\left\|\nabla \boldsymbol{\xi}_{h}^{n+\frac{1}{2}}\right\|^{2} \\
\leq \exp \left(\frac{2 C \Delta t}{\nu h^{2}} \sum_{n=0}^{N-1}\left\|\nabla \boldsymbol{\eta}^{n+\frac{1}{2}}\right\|^{2}\right)\left(\frac{2 C \Delta t}{\nu} \sum_{n=0}^{N-1}\left\|\nabla \boldsymbol{\eta}^{n}\right\|^{2}\left\|\nabla \boldsymbol{\eta}^{n+\frac{1}{2}}\right\|^{2}\right. \\
\left.+\frac{2 C \Delta t}{\nu} \sum_{n=0}^{N-1}\left\|\mathbf{f}\left(t_{n+\frac{1}{2}}\right)\right\|^{2}+\left\|\boldsymbol{\xi}_{h}^{0}\right\|^{2}\right) .
\end{gathered}
$$


Since $\|\nabla \boldsymbol{\eta}(t)\|$ is upper bounded by $C_{\boldsymbol{\eta}}$, we have

$$
\begin{aligned}
& \left\|\boldsymbol{\xi}_{h}^{N}\right\|^{2}+\nu \Delta t \sum_{n=0}^{N-1}\left\|\nabla \boldsymbol{\xi}_{h}^{n+\frac{1}{2}}\right\|^{2} \\
\leq & \exp \left(\frac{2 C C_{\boldsymbol{\eta}}^{2} T}{\nu h^{2}}\right)\left(\frac{2 C C_{\boldsymbol{\eta}}^{4} T}{\nu}+\frac{2 C \Delta t}{\nu} \sum_{n=0}^{N-1}\left\|\mathbf{f}\left(t_{n+\frac{1}{2}}\right)\right\|^{2}+\left\|\boldsymbol{\xi}_{h}^{0}\right\|^{2}\right) .
\end{aligned}
$$

This concludes the stability of scheme (4.1) for the modified Navier-Stokes equations with finite time interval.

Theorem 4.2 (Convergence). Under above sufficient assumptions, for a given sample $\omega \in \Omega$, let $\boldsymbol{\xi}\left(t_{n}\right)$ and $\boldsymbol{\xi}_{h}^{n}$ be pathwise solutions of (2.2) and (4.1), respectively. Assume that $\mathbf{u}_{0}(\omega) \in \mathbf{H}_{0}^{1}(D) \cap \dot{\mathbf{H}}^{\beta}(D),\|\nabla \mathbf{u}\|_{\infty}=\max _{t \in[0, T]}\|\nabla \mathbf{u}(t)\|$ and $\left\|\nabla \mathbf{u}_{h}\right\|_{\infty}=$ $\max _{t \in[0, T]}\left\|\nabla \mathbf{u}_{h}(t)\right\|$, there exists a constant $C$ independent of mesh size $h$ and time step $\Delta t$ such that

$$
\begin{aligned}
\left\|\boldsymbol{\xi}\left(t_{N}\right)-\boldsymbol{\xi}_{h}^{N}\right\|^{2}+\nu \Delta t \sum_{n=0}^{N-1}\left\|\nabla\left(\boldsymbol{\xi}^{n+\frac{1}{2}}-\boldsymbol{\xi}_{h}^{n+\frac{1}{2}}\right)\right\|^{2} \\
\leq C \exp \left(\frac{C T}{\nu h^{2}}\left\|\nabla \mathbf{u}_{h}\right\|_{\infty}^{2}\right)\left(\frac{T}{\nu}\|\nabla \mathbf{u}\|_{\infty}^{2} h^{2 \beta}\|\boldsymbol{\xi}\|_{\beta+1}^{2}\right. \\
+\frac{(\Delta t)^{2}}{2 \nu}\|\nabla \mathbf{u}\|_{\infty}^{2} \int_{t_{0}}^{t_{N}}\left\|\nabla \boldsymbol{\xi}_{t}(\tau)\right\|^{2} \mathrm{~d} \tau+\frac{T}{\nu}\|\nabla \mathbf{u}\|_{\infty}^{2}[\nabla \boldsymbol{\eta}]_{\alpha}^{2}|\Delta t|^{2 \alpha} \\
+\frac{(\Delta t)^{2}}{\nu}\left\|\nabla \mathbf{u}_{h}\right\|_{\infty}^{2} \int_{t_{0}}^{t_{N}}\left\|\nabla \boldsymbol{\xi}_{t}(\tau)\right\|^{2} \mathrm{~d} \tau+\frac{T}{\nu}\left\|\nabla \mathbf{u}_{h}\right\|_{\infty}^{2}[\nabla \boldsymbol{\eta}]_{\alpha}^{2}|\Delta t|^{2 \alpha} \\
+\frac{T}{\nu}\left\|\nabla \mathbf{u}_{h}\right\|_{\infty}^{2} h^{2 \beta}\|\boldsymbol{\xi}\|_{\beta+1}^{2}+\frac{h^{2 \beta+2}}{\nu} \int_{t_{0}}^{t_{N}}\left\|\boldsymbol{\xi}_{t}\right\|_{\beta+1}^{2} \mathrm{~d} \tau+\nu T h^{2 \beta}\|\boldsymbol{\xi}\|_{\beta+1}^{2} \\
+\frac{T}{\nu} h^{2 \beta}\|\boldsymbol{\eta}\|_{\beta}^{2}+\frac{(\Delta t)^{2}}{\nu} \int_{t_{0}}^{t_{N}}\left\|\pi_{t}(t)\right\|^{2} \mathrm{~d} \tau+\frac{T}{\nu}\left[\boldsymbol{\xi}_{t}\right]_{\alpha}^{2}(\Delta t)^{2 \alpha} \\
\left.+\nu(\Delta t)^{2} \int_{t_{0}}^{t_{N}}\left\|\nabla \boldsymbol{\xi}_{t}(\tau)\right\|^{2} \mathrm{~d} \tau+\left\|\mathbf{e}_{h}^{0}\right\|^{2}\right)+C h^{2 \beta+2}\|\boldsymbol{\xi}\|_{\beta+1}^{2}+\nu C T h^{2 \beta}\|\boldsymbol{\xi}\|_{\beta+1}^{2} .
\end{aligned}
$$

Proof. For $\mathbf{v}_{h} \in \mathbf{V}_{h}$, the continuous equation in (2.2) at $t=t_{n+\frac{1}{2}}$ satisfies

$$
\begin{gathered}
\left(\frac{\boldsymbol{\xi}\left(t_{n+1}\right)-\boldsymbol{\xi}\left(t_{n}\right)}{\Delta t}, \mathbf{v}_{h}\right)+\nu\left(\nabla \boldsymbol{\xi}^{n+\frac{1}{2}}, \nabla \mathbf{v}_{h}\right) \\
+\widetilde{c}\left(\mathbf{u}\left(t_{n+\frac{1}{2}}\right) ; \mathbf{u}\left(t_{n+\frac{1}{2}}\right), \mathbf{v}_{h}\right)-\left(\pi\left(t_{n+\frac{1}{2}}\right), \nabla \cdot \mathbf{v}_{h}\right) \\
=\left(\mathbf{f}\left(t_{n+\frac{1}{2}}\right), \mathbf{v}_{h}\right)+\left(\frac{\boldsymbol{\xi}\left(t_{n+1}\right)-\boldsymbol{\xi}\left(t_{n}\right)}{\Delta t}-\boldsymbol{\xi}_{t}\left(t_{n+\frac{1}{2}}\right), \mathbf{v}_{h}\right) \\
+\nu\left(\nabla \boldsymbol{\xi}^{n+\frac{1}{2}}-\nabla \boldsymbol{\xi}\left(t_{n+\frac{1}{2}}\right), \nabla \mathbf{v}_{h}\right) .
\end{gathered}
$$


Auxiliary Equations Approach for the Stochastic Unsteady Navier-Stokes Equations

Meanwhile, the discrete equation in (4.1) satisfies

$$
\begin{aligned}
& \left(\frac{\boldsymbol{\xi}_{h}^{n+1}-\boldsymbol{\xi}_{h}^{n}}{\Delta t}, \mathbf{v}_{h}\right)+\nu\left(\nabla \boldsymbol{\xi}_{h}^{n+\frac{1}{2}}, \nabla \mathbf{v}_{h}\right)+\widetilde{c}\left(\mathbf{u}_{h}^{n} ; \mathbf{u}_{h}^{n+\frac{1}{2}}, \mathbf{v}_{h}\right) \\
& -\left(\pi_{h}^{n+1}, \nabla \cdot \mathbf{v}_{h}\right)=\left(\mathbf{f}\left(t_{n+\frac{1}{2}}\right), \mathbf{v}_{h}\right)
\end{aligned}
$$

Further, we set the errors between the true, numerical, interpolation solutions for velocity and pressure as follows,

$$
\begin{aligned}
& \mathbf{e}^{n}:=\boldsymbol{\xi}^{n}-\boldsymbol{\xi}_{h}^{n}=\left(\boldsymbol{\xi}^{n}-I_{h} \boldsymbol{\xi}^{n}\right)+\left(I_{h} \boldsymbol{\xi}^{n}-\boldsymbol{\xi}_{h}^{n}\right)=\mathbf{e}_{I}^{n}+\mathbf{e}_{h}^{n}, \\
& z^{n}:=\pi^{n}-\pi_{h}^{n}=\left(\pi^{n}-J_{h} \pi^{n}\right)+\left(J_{h} \pi^{n}-\pi_{h}^{n}\right)=z_{J}^{n}+z_{h}^{n} .
\end{aligned}
$$

Hence, subtracting (4.3) from (4.2) follows the corresponding error equation

$$
\begin{aligned}
& \left(\frac{\mathbf{e}_{h}^{n+1}-\mathbf{e}_{h}^{n}}{\Delta t}, \mathbf{v}_{h}\right)+\nu\left(\nabla \mathbf{e}_{h}^{n+\frac{1}{2}}, \nabla \mathbf{v}_{h}\right)+\widetilde{c}\left(\mathbf{u}\left(t_{n+\frac{1}{2}}\right) ; \mathbf{u}\left(t_{n+\frac{1}{2}}\right), \mathbf{v}_{h}\right) \\
=- & \frac{\left(\mathbf{e}_{I}^{n+1}-\mathbf{e}_{I}^{n} ; \mathbf{u}_{h}^{n+\frac{1}{2}}, \mathbf{v}_{h}\right)-\left(z_{h}^{n+1}, \nabla \cdot \mathbf{v}_{h}\right)}{\Delta t}, \nu\left(\nabla \mathbf{e}_{I}^{n+\frac{1}{2}}, \nabla \mathbf{v}_{h}\right) \\
& +\left(z_{J}^{n+1}, \nabla \cdot \mathbf{v}_{h}\right)-\left(\pi\left(t_{n+1}\right)-\pi\left(t_{n+\frac{1}{2}}\right), \nabla \cdot \mathbf{v}_{h}\right) \\
& +\left(\frac{\boldsymbol{\xi}\left(t_{n+1}\right)-\boldsymbol{\xi}\left(t_{n}\right)}{\Delta t}-\boldsymbol{\xi}_{t}\left(t_{n+\frac{1}{2}}\right), \mathbf{v}_{h}\right)+\nu\left(\nabla \boldsymbol{\xi}^{n+\frac{1}{2}}-\nabla \boldsymbol{\xi}\left(t_{n+\frac{1}{2}}\right), \nabla \mathbf{v}_{h}\right) .
\end{aligned}
$$

Taking

$$
\mathbf{v}_{h}=2 \Delta t \mathbf{e}_{h}^{n+\frac{1}{2}}=\Delta t\left(\mathbf{e}_{h}^{n+1}+\mathbf{e}_{h}^{n}\right) \in \mathbf{V}_{h}
$$

we obtain

$$
\begin{aligned}
\left\|\mathbf{e}_{h}^{n+1}\right\|^{2}-\left\|\mathbf{e}_{h}^{n}\right\|^{2}+\left.2 \nu \Delta t\left\|\nabla \mathbf{e}_{h}^{n+\frac{1}{2}}\right\|\right|^{2} \\
=-2 \Delta t \widetilde{c}\left(\mathbf{u}\left(t_{n+\frac{1}{2}}\right) ; \mathbf{u}\left(t_{n+\frac{1}{2}}\right), \mathbf{e}_{h}^{n+\frac{1}{2}}\right)+2 \Delta t \widetilde{c}\left(\mathbf{u}_{h}^{n} ; \mathbf{u}_{h}^{n+\frac{1}{2}}, \mathbf{e}_{h}^{n+\frac{1}{2}}\right) \\
\quad-2\left(\mathbf{e}_{I}^{n+1}-\mathbf{e}_{I}^{n}, \mathbf{e}_{h}^{n+\frac{1}{2}}\right) \\
\quad-2 \nu \Delta t\left(\nabla \mathbf{e}_{I}^{n+\frac{1}{2}}, \nabla \mathbf{e}_{h}^{n+\frac{1}{2}}\right)+2 \Delta t\left(z_{J}^{n+1}, \nabla \cdot \mathbf{e}_{h}^{n+\frac{1}{2}}\right) \\
\quad-2 \Delta t\left(\pi\left(t_{n+1}\right)-\pi\left(t_{n+\frac{1}{2}}\right), \nabla \cdot \mathbf{e}_{h}^{n+\frac{1}{2}}\right) \\
\quad+2\left(\left(\boldsymbol{\xi}\left(t_{n+1}\right)-\boldsymbol{\xi}\left(t_{n}\right)\right)-\Delta t \boldsymbol{\xi}_{t}\left(t_{n+\frac{1}{2}}\right), \mathbf{e}_{h}^{n+\frac{1}{2}}\right) \\
\quad+\nu \Delta t\left(\nabla \boldsymbol{\xi}\left(t_{n+1}\right)+\nabla \boldsymbol{\xi}\left(t_{n}\right)-2 \nabla \boldsymbol{\xi}\left(t_{n+\frac{1}{2}}\right), \nabla \mathbf{e}_{h}^{n+\frac{1}{2}}\right)
\end{aligned}
$$


where $\left(z_{h}^{n+1}, \nabla \cdot \mathbf{v}_{h}\right)=0$ for $z_{h}^{n+1} \in Q_{h}$. The reminder of the following part is to bound each term in the above right hand side. For the nonlinear terms, by adding and subtracting $\widetilde{c}\left(\mathbf{u}\left(t_{n+\frac{1}{2}}\right) ; \mathbf{u}_{h}^{n+\frac{1}{2}}, \mathbf{e}_{h}^{n+\frac{1}{2}}\right)$, we have

$$
\begin{aligned}
& -\widetilde{c}\left(\mathbf{u}\left(t_{n+\frac{1}{2}}\right) ; \mathbf{u}\left(t_{n+\frac{1}{2}}\right), \mathbf{e}_{h}^{n+\frac{1}{2}}\right)+\widetilde{c}\left(\mathbf{u}_{h}^{n} ; \mathbf{u}_{h}^{n+\frac{1}{2}}, \mathbf{e}_{h}^{n+\frac{1}{2}}\right) \\
= & -\widetilde{c}\left(\mathbf{u}\left(t_{n+\frac{1}{2}}\right) ; \mathbf{u}\left(t_{n+\frac{1}{2}}\right), \mathbf{e}_{h}^{n+\frac{1}{2}}\right)+\widetilde{c}\left(\mathbf{u}\left(t_{n+\frac{1}{2}}\right) ; \mathbf{u}_{h}^{n+\frac{1}{2}}, \mathbf{e}_{h}^{n+\frac{1}{2}}\right) \\
& \quad-\widetilde{c}\left(\mathbf{u}\left(t_{n+\frac{1}{2}}\right) ; \mathbf{u}_{h}^{n+\frac{1}{2}}, \mathbf{e}_{h}^{n+\frac{1}{2}}\right)+\widetilde{c}\left(\mathbf{u}_{h}^{n} ; \mathbf{u}_{h}^{n+\frac{1}{2}}, \mathbf{e}_{h}^{n+\frac{1}{2}}\right) \\
& \quad-\widetilde{c}\left(\mathbf{u}\left(t_{n+\frac{1}{2}}\right) ; \mathbf{u}\left(t_{n+\frac{1}{2}}\right)-\mathbf{u}_{h}^{n+\frac{1}{2}}, \mathbf{e}_{h}^{n+\frac{1}{2}}\right)-\widetilde{c}\left(\mathbf{u}\left(t_{n+\frac{1}{2}}\right)-\mathbf{u}_{h}^{n} ; \mathbf{u}_{h}^{n+\frac{1}{2}}, \mathbf{e}_{h}^{n+\frac{1}{2}}\right) \\
= & :-I_{1}-I_{2} .
\end{aligned}
$$

Reorganizing the term $\mathbf{u}\left(t_{n+\frac{1}{2}}\right)-\mathbf{u}_{h}^{n+\frac{1}{2}}$ gives

$$
\begin{aligned}
& \mathbf{u}\left(t_{n+\frac{1}{2}}\right)-\mathbf{u}_{h}^{n+\frac{1}{2}} \\
= & \boldsymbol{\xi}\left(t_{n+\frac{1}{2}}\right)+\boldsymbol{\eta}\left(t_{n+\frac{1}{2}}\right)-\frac{1}{2}\left(\boldsymbol{\xi}_{h}^{n+1}+\boldsymbol{\eta}^{n+1}+\boldsymbol{\xi}_{h}^{n}+\boldsymbol{\eta}^{n}\right) \\
= & \frac{\boldsymbol{\xi}^{n+1}+\boldsymbol{\xi}^{n}}{2}-\frac{I_{h} \boldsymbol{\xi}^{n+1}+I_{h} \boldsymbol{\xi}^{n}}{2}+\frac{I_{h} \boldsymbol{\xi}^{n+1}+I_{h} \boldsymbol{\xi}^{n}}{2}-\frac{\boldsymbol{\xi}_{h}^{n+1}+\boldsymbol{\xi}_{h}^{n}}{2} \\
& \quad+\boldsymbol{\xi}\left(t_{n+\frac{1}{2}}\right)-\frac{\boldsymbol{\xi}^{n+1}+\boldsymbol{\xi}^{n}}{2}+\boldsymbol{\eta}\left(t_{n+\frac{1}{2}}\right)-\frac{\boldsymbol{\eta}^{n+1}+\boldsymbol{\eta}^{n}}{2} \\
& =\frac{1}{2}\left(\mathbf{e}_{I}^{n}+\mathbf{e}_{I}^{n+1}\right)+\frac{1}{2}\left(\mathbf{e}_{h}^{n}+\mathbf{e}_{h}^{n+1}\right)+\boldsymbol{\xi}\left(t_{n+\frac{1}{2}}\right)-\frac{\boldsymbol{\xi}^{n+1}+\boldsymbol{\xi}^{n}}{2}+\boldsymbol{\eta}\left(t_{n+\frac{1}{2}}\right)-\frac{\boldsymbol{\eta}^{n+1}+\boldsymbol{\eta}^{n}}{2} \\
= & \mathbf{e}_{I}^{n+\frac{1}{2}}+\mathbf{e}_{h}^{n+\frac{1}{2}}+\boldsymbol{\xi}\left(t_{n+\frac{1}{2}}\right)-\boldsymbol{\xi}^{n+\frac{1}{2}}+\boldsymbol{\eta}\left(t_{n+\frac{1}{2}}\right)-\boldsymbol{\eta}^{n+\frac{1}{2}}
\end{aligned}
$$

The first nonlinear term is estimated as follows

$$
\begin{aligned}
& I_{1}=\widetilde{c}\left(\mathbf{u}\left(t_{n+\frac{1}{2}}\right) ; \mathbf{u}\left(t_{n+\frac{1}{2}}\right)-\mathbf{u}_{h}^{n+\frac{1}{2}}, \mathbf{e}_{h}^{n+\frac{1}{2}}\right)=\widetilde{c}\left(\mathbf{u}\left(t_{n+\frac{1}{2}}\right) ; \mathbf{e}_{I}^{n+\frac{1}{2}}, \mathbf{e}_{h}^{n+\frac{1}{2}}\right) \\
& \quad+\widetilde{c}\left(\mathbf{u}\left(t_{n+\frac{1}{2}}\right) ; \boldsymbol{\xi}\left(t_{n+\frac{1}{2}}\right)-\boldsymbol{\xi}^{n+\frac{1}{2}}, \mathbf{e}_{h}^{n+\frac{1}{2}}\right)+\widetilde{c}\left(\mathbf{u}\left(t_{n+\frac{1}{2}}\right) ; \boldsymbol{\eta}\left(t_{n+\frac{1}{2}}\right)-\boldsymbol{\eta}^{n+\frac{1}{2}}, \mathbf{e}_{h}^{n+\frac{1}{2}}\right) \\
& =: I_{1,1}+I_{1,2}+I_{1,3} .
\end{aligned}
$$

For each subpart, we have

$$
\begin{aligned}
I_{1,1} & =\widetilde{c}\left(\mathbf{u}\left(t_{n+\frac{1}{2}}\right) ; \mathbf{e}_{I}^{n+\frac{1}{2}}, \mathbf{e}_{h}^{n+\frac{1}{2}}\right) \\
& \leq C\left\|\nabla \mathbf{u}\left(t_{n+\frac{1}{2}}\right)\right\|\left\|\nabla \mathbf{e}_{I}^{n+\frac{1}{2}}\right\|\left\|\nabla \mathbf{e}_{h}^{n+\frac{1}{2}}\right\| \\
& \leq \frac{\nu}{26}\left\|\nabla \mathbf{e}_{h}^{n+\frac{1}{2}}\right\|^{2}+\frac{C}{\nu}\left\|\nabla \mathbf{u}\left(t_{n+\frac{1}{2}}\right)\right\|^{2}\left\|\nabla \mathbf{e}_{I}^{n+\frac{1}{2}}\right\|^{2},
\end{aligned}
$$


Auxiliary Equations Approach for the Stochastic Unsteady Navier-Stokes Equations

$$
\begin{aligned}
I_{1,2} & =\widetilde{c}\left(\mathbf{u}\left(t_{n+\frac{1}{2}}\right) ; \boldsymbol{\xi}\left(t_{n+\frac{1}{2}}\right)-\boldsymbol{\xi}^{n+\frac{1}{2}}, \mathbf{e}_{h}^{n+\frac{1}{2}}\right) \\
& \leq C\left\|\nabla \mathbf{u}\left(t_{n+\frac{1}{2}}\right)\right\|\left\|\nabla\left(\boldsymbol{\xi}\left(t_{n+\frac{1}{2}}\right)-\boldsymbol{\xi}^{n+\frac{1}{2}}\right)\right\|\left\|\nabla \mathbf{e}_{h}^{n+\frac{1}{2}}\right\| \\
& \leq \frac{\nu}{26}\left\|\nabla \mathbf{e}_{h}^{n+\frac{1}{2}}\right\|^{2}+\frac{C}{\nu}\left\|\nabla \mathbf{u}\left(t_{n+\frac{1}{2}}\right)\right\|^{2}\left\|\nabla\left(\boldsymbol{\xi}\left(t_{n+\frac{1}{2}}\right)-\boldsymbol{\xi}^{n+\frac{1}{2}}\right)\right\|^{2}, \\
I_{1,3} & =\widetilde{c}\left(\mathbf{u}\left(t_{n+\frac{1}{2}}\right) ; \boldsymbol{\eta}\left(t_{n+\frac{1}{2}}\right)-\boldsymbol{\eta}^{n+\frac{1}{2}}, \mathbf{e}_{h}^{n+\frac{1}{2}}\right) \\
& \leq C\left\|\nabla \mathbf{u}\left(t_{n+\frac{1}{2}}\right)\right\|\left\|\nabla\left(\boldsymbol{\eta}\left(t_{n+\frac{1}{2}}\right)-\boldsymbol{\eta}^{n+\frac{1}{2}}\right)\right\|\left\|\mathbf{e}_{h}^{n+\frac{1}{2}}\right\| \\
& \leq \frac{\nu}{26}\left\|\nabla \mathbf{e}_{h}^{n+\frac{1}{2}}\right\|^{2}+\frac{C}{\nu}\left\|\nabla \mathbf{u}\left(t_{n+\frac{1}{2}}\right)\right\|^{2}\left\|\nabla\left(\boldsymbol{\eta}\left(t_{n+\frac{1}{2}}\right)-\boldsymbol{\eta}^{n+\frac{1}{2}}\right)\right\|^{2} .
\end{aligned}
$$

The two terms

$$
\left\|\nabla\left(\boldsymbol{\xi}\left(t_{n+\frac{1}{2}}\right)-\boldsymbol{\xi}^{n+\frac{1}{2}}\right)\right\|^{2} \quad \text { and } \quad\left\|\nabla\left(\boldsymbol{\eta}\left(t_{n+\frac{1}{2}}\right)-\boldsymbol{\eta}^{n+\frac{1}{2}}\right)\right\|^{2}
$$

above are estimated as follows. From the Newton-Leibniz formula, we have

$$
\boldsymbol{\xi}\left(t_{n+1}\right)+\boldsymbol{\xi}\left(t_{n}\right)-2 \boldsymbol{\xi}\left(t_{n+\frac{1}{2}}\right)=\int_{t_{n+\frac{1}{2}}}^{t_{n+1}} \boldsymbol{\xi}_{t}(\tau) \mathrm{d} \tau-\int_{t_{n}}^{t_{n+\frac{1}{2}}} \boldsymbol{\xi}_{t}(\tau) \mathrm{d} \tau
$$

With the Cauchy-Schwarz inequality in time direction, taking gradient of the above equation in space on both sides gives

$$
\begin{aligned}
& \left\|\nabla\left(\boldsymbol{\xi}\left(t_{n+1}\right)+\boldsymbol{\xi}\left(t_{n}\right)-2 \boldsymbol{\xi}\left(t_{n+\frac{1}{2}}\right)\right)\right\|^{2} \\
\leq & \left\|(\Delta t)^{1 / 2}\left(\int_{t_{n}}^{t_{n+1}}\left|\nabla \boldsymbol{\xi}_{t}(\tau)\right|^{2} \mathrm{~d} \tau\right)^{1 / 2}\right\|^{2} \\
= & \Delta t \int_{t_{n}}^{t_{n+1}}\left\|\nabla \boldsymbol{\xi}_{t}(\tau)\right\|^{2} \mathrm{~d} \tau .
\end{aligned}
$$

It leads to

$$
\left\|\nabla\left(\boldsymbol{\xi}\left(t_{n+\frac{1}{2}}\right)-\boldsymbol{\xi}^{n+\frac{1}{2}}\right)\right\|^{2} \leq \frac{\Delta t}{4} \int_{t_{n}}^{t_{n+1}}\left\|\nabla \boldsymbol{\xi}_{t}(\tau)\right\|^{2} \mathrm{~d} \tau
$$

Since we have assumed that $\boldsymbol{\eta}(t)$ is $\alpha$-hölder continuous in time, we set

$$
[\boldsymbol{\eta}]_{\alpha}=\sup \left\{\frac{|\boldsymbol{\eta}(t, x)-\boldsymbol{\eta}(s, x)|}{|t-s|^{\alpha}}: t, s \in[0, T] \text {, and } s \neq t, a . s . x \in D\right\},
$$

similar for $\boldsymbol{\xi}$. Then, it follows

$$
\begin{aligned}
& \left|\boldsymbol{\eta}\left(t_{n+\frac{1}{2}}\right)-\boldsymbol{\eta}^{n+\frac{1}{2}}\right| \\
\leq & \frac{1}{2}\left(\left|\boldsymbol{\eta}\left(t_{n+1}\right)-\boldsymbol{\eta}\left(t_{n+\frac{1}{2}}\right)\right|+\left|\boldsymbol{\eta}\left(t_{n+\frac{1}{2}}\right)-\boldsymbol{\eta}\left(t_{n}\right)\right|\right) \leq[\boldsymbol{\eta}]_{\alpha}\left(\frac{1}{2}\right)^{\alpha}|\Delta t|^{\alpha} .
\end{aligned}
$$


It follows that

$$
\begin{aligned}
\left\|\nabla\left(\boldsymbol{\eta}\left(t_{n+\frac{1}{2}}\right)-\boldsymbol{\eta}^{n+\frac{1}{2}}\right)\right\|^{2} & =\int_{D}\left|\nabla\left(\boldsymbol{\eta}\left(t_{n+\frac{1}{2}}\right)-\boldsymbol{\eta}^{n+\frac{1}{2}}\right)\right|^{2} \mathrm{~d} D \\
& \leq C 2^{-2 \alpha}[\nabla \boldsymbol{\eta}]_{\alpha}^{2}|\Delta t|^{2 \alpha} \leq C[\nabla \boldsymbol{\eta}]_{\alpha}^{2}|\Delta t|^{2 \alpha} .
\end{aligned}
$$

The second nonlinear term is estimated as follows

$$
\begin{aligned}
I_{2} & =\widetilde{c}\left(\mathbf{u}\left(t_{n+\frac{1}{2}}\right)-\mathbf{u}_{h}^{n} ; \mathbf{u}_{h}^{n+\frac{1}{2}}, \mathbf{e}_{h}^{n+\frac{1}{2}}\right) \\
& =\widetilde{c}\left(\mathbf{u}\left(t_{n+\frac{1}{2}}\right)-\mathbf{u}\left(t_{n}\right) ; \mathbf{u}_{h}^{n+\frac{1}{2}}, \mathbf{e}_{h}^{n+\frac{1}{2}}\right)+\widetilde{c}\left(\mathbf{u}\left(t_{n}\right)-\mathbf{u}_{h}^{n} ; \mathbf{u}_{h}^{n+\frac{1}{2}}, \mathbf{e}_{h}^{n+\frac{1}{2}}\right) \\
& =\widetilde{c}\left(\mathbf{u}\left(t_{n+\frac{1}{2}}\right)-\mathbf{u}\left(t_{n}\right) ; \mathbf{u}_{h}^{n+\frac{1}{2}}, \mathbf{e}_{h}^{n+\frac{1}{2}}\right)+\widetilde{c}\left(\mathbf{e}_{h}^{n} ; \mathbf{u}_{h}^{n+\frac{1}{2}}, \mathbf{e}_{h}^{n+\frac{1}{2}}\right)+\widetilde{c}\left(\mathbf{e}_{I}^{n} ; \mathbf{u}_{h}^{n+\frac{1}{2}}, \mathbf{e}_{h}^{n+\frac{1}{2}}\right) \\
& =: I_{2,1}+I_{2,2}+I_{2,3} .
\end{aligned}
$$

For the sub-terms, we have

$$
\begin{aligned}
I_{2,1}= & \widetilde{c}\left(\mathbf{u}\left(t_{n+\frac{1}{2}}\right)-\mathbf{u}\left(t_{n}\right) ; \mathbf{u}_{h}^{n+\frac{1}{2}}, \mathbf{e}_{h}^{n+\frac{1}{2}}\right) \\
& =\widetilde{c}\left(\boldsymbol{\xi}\left(t_{n+\frac{1}{2}}\right)-\boldsymbol{\xi}\left(t_{n}\right) ; \mathbf{u}_{h}^{n+\frac{1}{2}}, \mathbf{e}_{h}^{n+\frac{1}{2}}\right)+\widetilde{c}\left(\boldsymbol{\eta}\left(t_{n+\frac{1}{2}}\right)-\boldsymbol{\eta}\left(t_{n}\right) ; \mathbf{u}_{h}^{n+\frac{1}{2}}, \mathbf{e}_{h}^{n+\frac{1}{2}}\right) \\
& =I_{2,1,1}+I_{2,1,2}, \\
I_{2,1,1} & =\widetilde{c}\left(\boldsymbol{\xi}\left(t_{n+\frac{1}{2}}\right)-\boldsymbol{\xi}\left(t_{n}\right) ; \mathbf{u}_{h}^{n+\frac{1}{2}}, \mathbf{e}_{h}^{n+\frac{1}{2}}\right) \\
& \leq C\left\|\nabla\left(\boldsymbol{\xi}\left(t_{n+\frac{1}{2}}\right)-\boldsymbol{\xi}\left(t_{n}\right)\right)\right\|\left\|\nabla \mathbf{u}_{h}^{n+\frac{1}{2}}\right\|\left\|\nabla \mathbf{e}_{h}^{n+\frac{1}{2}}\right\| \\
& \leq \frac{\nu}{26}\left\|\nabla \mathbf{e}_{h}^{n+\frac{1}{2}}\right\|^{2}+\frac{C}{\nu}\left\|\nabla \mathbf{u}_{h}^{n+\frac{1}{2}}\right\|^{2}\left\|\nabla\left(\boldsymbol{\xi}\left(t_{n+\frac{1}{2}}\right)-\boldsymbol{\xi}\left(t_{n}\right)\right)\right\|^{2} \\
I_{2,1,2} & =\widetilde{c}\left(\boldsymbol{\eta}\left(t_{n+\frac{1}{2}}\right)-\boldsymbol{\eta}\left(t_{n}\right) ; \mathbf{u}_{h}^{n+\frac{1}{2}}, \mathbf{e}_{h}^{n+\frac{1}{2}}\right) \\
& \leq C\left\|\nabla\left(\boldsymbol{\eta}\left(t_{n+\frac{1}{2}}\right)-\boldsymbol{\eta}\left(t_{n}\right)\right)\right\|\left\|\nabla \mathbf{u}_{h}^{n+\frac{1}{2}}\right\|\left\|\nabla \mathbf{e}_{h}^{n+\frac{1}{2}}\right\| \\
& \leq \frac{\nu}{26}\left\|\nabla \mathbf{e}_{h}^{n+\frac{1}{2}}\right\|\left\|^{2}+\frac{C}{\nu}\right\| \nabla \mathbf{u}_{h}^{n+\frac{1}{2}}\left\|^{2}\right\| \nabla\left(\boldsymbol{\eta}\left(t_{n+\frac{1}{2}}\right)-\boldsymbol{\eta}\left(t_{n}\right)\right) \|^{2} .
\end{aligned}
$$

For the terms associated to $\boldsymbol{\xi}$ and $\boldsymbol{\eta}$, we have the following estimates,

$$
\begin{aligned}
& \boldsymbol{\xi}\left(t_{n+\frac{1}{2}}\right)-\boldsymbol{\xi}\left(t_{n}\right)=\int_{t_{n}}^{t_{n+\frac{1}{2}}} \boldsymbol{\xi}_{t}(\tau) \mathrm{d} \tau \\
& \left\|\nabla \boldsymbol{\xi}\left(t_{n+\frac{1}{2}}\right)-\nabla \boldsymbol{\xi}\left(t_{n}\right)\right\|^{2} \leq \Delta t \int_{t_{n}}^{t_{n+1}}\left\|\nabla \boldsymbol{\xi}_{t}(\tau)\right\|^{2} \mathrm{~d} \tau \\
& \left|\boldsymbol{\eta}\left(t_{n+\frac{1}{2}}\right)-\boldsymbol{\eta}\left(t_{n}\right)\right| \leq[\boldsymbol{\eta}]_{\alpha}\left(\frac{1}{2}\right)^{\alpha}|\Delta t|^{\alpha},
\end{aligned}
$$


Auxiliary Equations Approach for the Stochastic Unsteady Navier-Stokes Equations

$$
\begin{aligned}
& \left\|\nabla \boldsymbol{\eta}\left(t_{n+\frac{1}{2}}\right)-\nabla \boldsymbol{\eta}\left(t_{n}\right)\right\|^{2} \leq C[\nabla \boldsymbol{\eta}]_{\alpha}^{2}\left(\frac{1}{2}\right)^{2 \alpha}|\Delta t|^{2 \alpha} \leq C[\nabla \boldsymbol{\eta}]_{\alpha}^{2}|\Delta t|^{2 \alpha}, \\
& I_{2,2}=\widetilde{c}\left(\mathbf{e}_{h}^{n} ; \mathbf{u}_{h}^{n+\frac{1}{2}}, \mathbf{e}_{h}^{n+\frac{1}{2}}\right) \leq C\left\|\nabla \mathbf{e}_{h}^{n}\right\|\left\|\nabla \mathbf{u}_{h}^{n+\frac{1}{2}}\right\|\left\|\nabla \mathbf{e}_{h}^{n+\frac{1}{2}}\right\| \\
& \quad \leq \frac{\nu}{26}\left\|\nabla \mathbf{e}_{h}^{n+\frac{1}{2}}\right\|^{2}+\frac{C}{\nu}\left\|\nabla \mathbf{u}_{h}^{n+\frac{1}{2}}\right\|^{2}\left\|\nabla \mathbf{e}_{h}^{n}\right\|^{2} \\
& \quad \leq \frac{\nu}{26}\left\|\nabla \mathbf{e}_{h}^{n+\frac{1}{2}}\right\|^{2}+\frac{C}{\nu h^{2}}\left\|\nabla \mathbf{u}_{h}^{n+\frac{1}{2}}\right\|^{2}\left\|\mathbf{e}_{h}^{n}\right\|^{2}, \\
& I_{2,3}=\widetilde{c}\left(\mathbf{e}_{I}^{n} ; \mathbf{u}_{h}^{n+\frac{1}{2}}, \mathbf{e}_{h}^{n+\frac{1}{2}}\right) \leq C\left\|\nabla \mathbf{e}_{I}^{n}\right\|\left\|_{h}^{n+\frac{1}{2}}\right\|\left\|\mathbf{u}_{h}^{n+\frac{1}{2}}\right\| \\
& \leq\left.\frac{\nu}{26}\left\|\nabla \mathbf{e}_{h}^{n+\frac{1}{2}}\right\|\right|^{2}+\frac{C}{\nu}\left\|\nabla \mathbf{u}_{h}^{n+\frac{1}{2}}\right\|\left\|^{2}\right\| \nabla \mathbf{e}_{I}^{n} \|^{2} .
\end{aligned}
$$

For the term associated with discrete derivative in time, we have

$$
\begin{aligned}
& \left(\mathbf{e}_{I}^{n+1}-\mathbf{e}_{I}^{n}, \mathbf{e}_{h}^{n+\frac{1}{2}}\right)=\left(\int_{t_{n}}^{t_{n+1}}\left(\mathbf{e}_{I}(\tau)\right)_{t} \mathrm{~d} \tau, \mathbf{e}_{h}^{n+\frac{1}{2}}\right) \\
\leq & \left\|\nabla \mathbf{e}_{h}^{n+\frac{1}{2}}\right\|\left\|\int_{t_{n}}^{t_{n+1}}\left(\mathbf{e}_{I}(\tau)\right)_{t} \mathrm{~d} \tau\right\| \\
\leq & \frac{\nu \Delta t}{26}\left\|\nabla \mathbf{e}_{h}^{n+\frac{1}{2}}\right\|^{2}+\frac{C}{\nu} \int_{t_{n}}^{t_{n+1}}\left\|\left(\mathbf{e}_{I}(\tau)\right)_{t}\right\|^{2} \mathrm{~d} \tau .
\end{aligned}
$$

For the terms of the Stokes interpolation operators, we have

$$
\begin{aligned}
& \nu\left(\nabla \mathbf{e}_{I}^{n+\frac{1}{2}}, \nabla \mathbf{e}_{h}^{n+\frac{1}{2}}\right) \leq \nu\left\|\nabla \mathbf{e}_{I}^{n+\frac{1}{2}}\right\|\left\|\nabla \mathbf{e}_{h}^{n+\frac{1}{2}}\right\| \leq \frac{\nu}{26}\left\|\nabla \mathbf{e}_{h}^{n+\frac{1}{2}}\right\|^{2}+C \nu\left\|\nabla \mathbf{e}_{I}^{n+\frac{1}{2}}\right\|^{2}, \\
& \left(z_{J}^{n+1}, \nabla \cdot \mathbf{e}_{h}^{n+\frac{1}{2}}\right) \leq\left\|z_{J}^{n+1}\right\|\left\|\nabla \cdot \mathbf{e}_{h}^{n+\frac{1}{2}}\right\| \leq\left.\frac{\nu}{26}\left\|\nabla \mathbf{e}_{h}^{n+\frac{1}{2}}\right\|\right|^{2}+\frac{C}{\nu}\left\|z_{J}^{n+1}\right\|^{2} .
\end{aligned}
$$

For the pressure term, we have

$$
\begin{aligned}
\left(\pi\left(t_{n+1}\right)-\pi\left(t_{n+\frac{1}{2}}\right), \nabla \cdot \mathbf{e}_{h}^{n+\frac{1}{2}}\right) & \leq\left\|\int_{t_{n}}^{t_{n+1}}\left|\pi_{t}(t)\right| \mathrm{d} \tau\right\|\left\|\nabla \cdot \mathbf{e}_{h}^{n+\frac{1}{2}}\right\| \\
& \leq \frac{\nu}{26}\left\|\nabla \mathbf{e}_{h}^{n+\frac{1}{2}}\right\|^{2}+\frac{C \Delta t}{\nu} \int_{t_{n}}^{t_{n+1}}\left\|\pi_{t}(t)\right\|^{2} \mathrm{~d} \tau .
\end{aligned}
$$

For the last two terms, from Newton-Leibniz formula, we have

$$
\boldsymbol{\xi}\left(t_{n+1}\right)-\boldsymbol{\xi}\left(t_{n}\right)-\Delta t \boldsymbol{\xi}_{t}\left(t_{n+\frac{1}{2}}\right)=\int_{t_{n}}^{t_{n+1}} \boldsymbol{\xi}_{t}(\tau)-\boldsymbol{\xi}_{t}\left(t_{n+\frac{1}{2}}\right) \mathrm{d} \tau
$$


It follows that

$$
\begin{aligned}
& \left(\left(\boldsymbol{\xi}\left(t_{n+1}\right)-\boldsymbol{\xi}\left(t_{n}\right)\right)-\Delta t \boldsymbol{\xi}_{t}\left(t_{n+\frac{1}{2}}\right), \mathbf{e}_{h}^{n+\frac{1}{2}}\right) \\
& =\left(\int_{t_{n}}^{t_{n+1}} \boldsymbol{\xi}_{t}(\tau)-\boldsymbol{\xi}_{t}\left(t_{n+\frac{1}{2}}\right) \mathrm{d} \tau, \mathbf{e}_{h}^{n+\frac{1}{2}}\right) \\
& \leq C\left\|\int_{t_{n}}^{t_{n+1}} \boldsymbol{\xi}_{t}(\tau)-\boldsymbol{\xi}_{t}\left(t_{n+\frac{1}{2}}\right) \mathrm{d} \tau\right\|\left\|\nabla \mathbf{e}_{h}^{n+\frac{1}{2}}\right\| \\
& \quad \leq \frac{\nu \Delta t}{26}\left\|\nabla \mathbf{e}_{h}^{n+\frac{1}{2}}\right\|^{2}+\frac{C}{\nu} \int_{t_{n}}^{t_{n+1}}\left\|\boldsymbol{\xi}_{t}(\tau)-\boldsymbol{\xi}_{t}\left(t_{n+\frac{1}{2}}\right)\right\|^{2} \mathrm{~d} \tau \\
& \quad \leq \frac{\nu \Delta t}{26}\left\|\nabla \mathbf{e}_{h}^{n+\frac{1}{2}}\right\|^{2}+\frac{C}{\nu} \Delta t\left[\boldsymbol{\xi}_{t}\right]_{\alpha}^{2}(\Delta t)^{2 \alpha}, \\
& \nu\left(\nabla \boldsymbol{\xi}\left(t_{n+1}\right)+\nabla \boldsymbol{\xi}\left(t_{n}\right)-2 \nabla \boldsymbol{\xi}\left(t_{n+\frac{1}{2}}\right), \nabla \mathbf{e}_{h}^{n+\frac{1}{2}}\right) \\
& \quad \leq \nu\left(\Delta t \int_{t_{n}}^{t_{n+1}}\left\|\nabla \boldsymbol{\xi}_{t}(\tau)\right\| \mathrm{d} \tau\right)^{\frac{1}{2}}\left\|\nabla \mathbf{e}_{h}^{n+\frac{1}{2}}\right\| \\
& \quad \leq \frac{2 \nu}{26}\left\|\nabla \mathbf{e}_{h}^{n+\frac{1}{2}}\right\|\left\|^{2}+\nu C \Delta t \int_{t_{n}}^{t_{n+1}}\right\| \nabla \boldsymbol{\xi}_{t}(\tau) \|^{2} \mathrm{~d} \tau
\end{aligned}
$$

Combing all the above inequalities together obtains

$$
\begin{aligned}
& \left\|\mathbf{e}_{h}^{n+1}\right\|^{2}-\left\|\mathbf{e}_{h}^{n}\right\|^{2}+\left.\nu \Delta t\left\|\nabla \mathbf{e}_{h}^{n+\frac{1}{2}}\right\|\right|^{2} \\
& \leq\left.\frac{2 C \Delta t}{\nu h^{2}}\left\|\nabla \mathbf{u}_{h}^{n+\frac{1}{2}}\right\|\right|^{2}\left\|\mathbf{e}_{h}^{n}\right\|^{2}+\frac{2 C \Delta t}{\nu}\left\|\nabla \mathbf{u}\left(t_{n+\frac{1}{2}}\right)\right\|^{2}\left\|\nabla \mathbf{e}_{I}^{n+\frac{1}{2}}\right\|^{2} \\
& \quad+\frac{C(\Delta t)^{2}}{2 \nu}\left\|\nabla \mathbf{u}\left(t_{n+\frac{1}{2}}\right)\right\|^{2} \int_{t_{n}}^{t_{n+1}}\left\|\nabla \boldsymbol{\xi}_{t}(\tau)\right\|^{2} \mathrm{~d} \tau+\frac{2 C \Delta t}{\nu}\left\|\nabla \mathbf{u}\left(t_{n+\frac{1}{2}}\right)\right\|^{2}[\nabla \boldsymbol{\eta}]_{\alpha}^{2}|\Delta t|^{2 \alpha} \\
& \quad+\frac{2 C(\Delta t)^{2}}{\nu}\left\|\nabla \mathbf{u}_{h}^{n+\frac{1}{2}}\right\|^{2} \int_{t_{n}}^{t_{n+1}}\left\|\nabla \boldsymbol{\xi}_{t}(\tau)\right\|^{2} \mathrm{~d} \tau+\frac{2 C \Delta t}{\nu}\left\|\nabla \mathbf{u}_{h}^{n+\frac{1}{2}}\right\|^{2}[\nabla \boldsymbol{\eta}]_{\alpha}^{2}|\Delta t|^{2 \alpha} \\
& +\frac{2 C \Delta t}{\nu}\left\|\nabla \mathbf{u}_{h}^{n+\frac{1}{2}}\right\|^{2}\left\|\nabla \mathbf{e}_{I}^{n}\right\|^{2}+\frac{2 C}{\nu} \int_{t_{n}}^{t_{n+1}}\left\|\left(\mathbf{e}_{I}(\tau)\right)_{t}\right\|^{2} \mathrm{~d} \tau \\
& +2 \nu C \Delta t\left\|\nabla \mathbf{e}_{I}^{n+\frac{1}{2}}\right\|^{2}+\frac{2 C \Delta t}{\nu}\left\|z_{J}^{n+1}\right\|^{2}+\frac{2 C(\Delta t)^{2}}{\nu} \int_{t_{n}}^{t_{n+1}}\left\|\pi_{t}(t)\right\|^{2} \mathrm{~d} \tau \\
& \quad+\frac{2 C}{\nu} \Delta t\left[\boldsymbol{\xi}_{t}\right]_{\alpha}^{2}(\Delta t)^{2 \alpha}+\nu C(\Delta t)^{2} \int_{t_{n}}^{t_{n+1}}\left\|\nabla \boldsymbol{\xi}_{t}(\tau)\right\|^{2} \mathrm{~d} \tau .
\end{aligned}
$$

Summing up the above inequalities from $n=0$ to $n=N-1$ gives

$$
\left\|\mathbf{e}_{h}^{N}\right\|^{2}-\left\|\mathbf{e}_{h}^{0}\right\|^{2}+\nu \Delta t \sum_{n=0}^{N-1}\left\|\nabla \mathbf{e}_{h}^{n+\frac{1}{2}}\right\|^{2}
$$


Auxiliary Equations Approach for the Stochastic Unsteady Navier-Stokes Equations

$$
\begin{aligned}
\leq \frac{2 C}{\nu h^{2}} & \left\|\nabla \mathbf{u}_{h}\right\|_{\infty}^{2} \sum_{n=0}^{N-1}\left\|\mathbf{e}_{h}^{n}\right\|^{2}+\frac{2 C \Delta t}{\nu}\|\nabla \mathbf{u}\|_{\infty}^{2} \sum_{n=0}^{N-1}\left\|\nabla \mathbf{e}_{I}^{n+\frac{1}{2}}\right\|^{2} \\
& +\frac{C(\Delta t)^{2}}{2 \nu}\|\nabla \mathbf{u}\|_{\infty}^{2} \int_{t_{0}}^{t_{N}}\left\|\nabla \boldsymbol{\xi}_{t}(\tau)\right\|^{2} \mathrm{~d} \tau+\frac{2 C(N \Delta t)}{\nu}\|\nabla \mathbf{u}\|_{\infty}^{2}[\nabla \boldsymbol{\eta}]_{\alpha}^{2}|\Delta t|^{2 \alpha} \\
& +\frac{2 C(\Delta t)^{2}}{\nu}\left\|\nabla \mathbf{u}_{h}\right\|_{\infty}^{2} \int_{t_{0}}^{t_{N}}\left\|\nabla \boldsymbol{\xi}_{t}(\tau)\right\|^{2} \mathrm{~d} \tau+\frac{2 C(N \Delta t)}{\nu}\left\|\nabla \mathbf{u}_{h}\right\|_{\infty}^{2}[\nabla \boldsymbol{\eta}]_{\alpha}^{2}|\Delta t|^{2 \alpha} \\
& +\frac{2 C \Delta t}{\nu}\left\|\nabla \mathbf{u}_{h}\right\|_{\infty}^{2} \sum_{n=0}^{N-1}\left\|\nabla \mathbf{e}_{I}^{n}\right\|^{2}+\frac{2 C}{\nu} \int_{t_{0}}^{t_{N}}\left\|\left(\mathbf{e}_{I}(\tau)\right)_{t}\right\|^{2} \mathrm{~d} \tau \\
& +2 \nu C \Delta t \sum_{n=0}^{N-1}\left\|\nabla \mathbf{e}_{I}^{n+\frac{1}{2}}\right\|^{2}+\frac{2 C \Delta t}{\nu} \sum_{n=0}^{N-1}\left\|z_{J}^{n+1}\right\|^{2}+\frac{2 C(\Delta t)^{2}}{\nu} \int_{t_{0}}^{t_{N}}\left\|\pi_{t}(t)\right\|^{2} \mathrm{~d} \tau \\
& +\frac{2 C}{\nu}(N \Delta t)\left[\boldsymbol{\xi}_{t}\right]_{\alpha}^{2}(\Delta t)^{2 \alpha}+\nu C(\Delta t)^{2} \int_{t_{0}}^{t_{N}}\left\|\nabla \boldsymbol{\xi}_{t}(\tau)\right\|^{2} \mathrm{~d} \tau
\end{aligned}
$$

Further, applying interpolation inequalities, we have

$$
\begin{aligned}
\left\|\mathbf{e}_{h}^{N}\right\|^{2}-\left\|\mathbf{e}_{h}^{0}\right\|^{2}+\nu \Delta t \sum_{n=0}^{N-1}\left\|\nabla \mathbf{e}_{h}^{n+\frac{1}{2}}\right\|^{2} \\
\leq \frac{2 C \Delta t}{\nu h^{2}}\left\|\nabla \mathbf{u}_{h}\right\|_{\infty}^{2} \sum_{n=0}^{N-1}\left\|\mathbf{e}_{h}^{n}\right\|^{2}+\frac{2 C N \Delta t}{\nu}\|\nabla \mathbf{u}\|_{\infty}^{2} h^{2 \beta}\|\boldsymbol{\xi}\|_{\beta+1}^{2} \\
\quad+\frac{C(\Delta t)^{2}}{2 \nu}\|\nabla \mathbf{u}\|_{\infty}^{2} \int_{t_{0}}^{t_{N}}\left\|\nabla \boldsymbol{\xi}_{t}(\tau)\right\|^{2} \mathrm{~d} \tau+\frac{2 C(N \Delta t)}{\nu}\|\nabla \mathbf{u}\|_{\infty}^{2}[\nabla \boldsymbol{\eta}]_{\alpha}^{2}|\Delta t|^{2 \alpha} \\
\quad+\frac{2 C(\Delta t)^{2}}{\nu}\left\|\nabla \mathbf{u}_{h}\right\|_{\infty}^{2} \int_{t_{0}}^{t_{N}}\left\|\nabla \boldsymbol{\xi}_{t}(\tau)\right\|^{2} \mathrm{~d} \tau+\frac{2 C(N \Delta t)}{\nu}\left\|\nabla \mathbf{u}_{h}\right\|_{\infty}^{2}[\nabla \boldsymbol{\eta}]_{\alpha}^{2}|\Delta t|^{2 \alpha} \\
\quad+\frac{2 C N \Delta t}{\nu}\left\|\nabla \mathbf{u}_{h}\right\|_{\infty}^{2} h^{2 \beta}\|\boldsymbol{\xi}\|_{\beta+1}^{2}+\frac{2 C}{\nu} h^{2 \beta+2} \int_{t_{0}}^{t_{N}}\left\|\boldsymbol{\xi}_{t}\right\|_{\beta+1}^{2} \mathrm{~d} \tau \\
\quad+2 \nu C N \Delta t h^{2 \beta}\|\boldsymbol{\xi}\|_{\beta+1}^{2}+\frac{2 C N \Delta t}{\nu} h^{2 \beta}\|\boldsymbol{\eta}\|_{\beta}^{2}+\frac{2 C(\Delta t)^{2}}{\nu} \int_{t_{0}}^{t_{N}}\left\|\pi_{t}(t)\right\|^{2} \mathrm{~d} \tau \\
\quad+\frac{2 C}{\nu}(N \Delta t)\left[\boldsymbol{\xi}_{t}\right]_{\alpha}^{2}(\Delta t)^{2 \alpha}+\nu C(\Delta t)^{2} \int_{t_{0}}^{t_{N}}\left\|\nabla \boldsymbol{\xi}_{t}(\tau)\right\|^{2} \mathrm{~d} \tau .
\end{aligned}
$$

By the discrete Gronwall inequality, we have

$$
\begin{aligned}
\left\|\mathbf{e}_{h}^{N}\right\|^{2}+\left.\nu \Delta t \sum_{n=0}^{N-1}\left\|\nabla \mathbf{e}_{h}^{n+\frac{1}{2}}\right\|\right|^{2} \\
\leq \exp \left(\frac{2 C N \Delta t}{\nu h^{2}}\left\|\nabla \mathbf{u}_{h}\right\|_{\infty}^{2}\right)\left(\frac{2 C N \Delta t}{\nu}\|\nabla \mathbf{u}\|_{\infty}^{2} h^{2 \beta}\|\boldsymbol{\xi}\|_{\beta+1}^{2}\right. \\
\quad+\frac{C(\Delta t)^{2}}{2 \nu}\|\nabla \mathbf{u}\|_{\infty}^{2} \int_{t_{0}}^{t_{N}}\left\|\nabla \boldsymbol{\xi}_{t}(\tau)\right\|^{2} \mathrm{~d} \tau+\frac{2 C(N \Delta t)}{\nu}\|\nabla \mathbf{u}\|_{\infty}^{2}[\nabla \boldsymbol{\eta}]_{\alpha}^{2}|\Delta t|^{2 \alpha}
\end{aligned}
$$




$$
\begin{aligned}
& +\frac{2 C(\Delta t)^{2}}{\nu}\left\|\nabla \mathbf{u}_{h}\right\|_{\infty}^{2} \int_{t_{0}}^{t_{N}}\left\|\nabla \boldsymbol{\xi}_{t}(\tau)\right\|^{2} \mathrm{~d} \tau+\frac{2 C(N \Delta t)}{\nu}\left\|\nabla \mathbf{u}_{h}\right\|_{\infty}^{2}[\nabla \boldsymbol{\eta}]_{\alpha}^{2}|\Delta t|^{2 \alpha} \\
& +\frac{2 C N \Delta t}{\nu}\left\|\nabla \mathbf{u}_{h}\right\|_{\infty}^{2} h^{2 \beta}\|\boldsymbol{\xi}\|_{\beta+1}^{2}+\frac{2 C}{\nu} h^{2 \beta+2} \int_{t_{0}}^{t_{N}}\left\|\boldsymbol{\xi}_{t}\right\|_{\beta+1}^{2} \mathrm{~d} \tau \\
& +2 \nu C N \Delta t h^{2 \beta}\|\boldsymbol{\xi}\|_{\beta+1}^{2}+\frac{2 C N \Delta t}{\nu} h^{2 \beta}\|\boldsymbol{\eta}\|_{\beta}^{2}+\frac{2 C(\Delta t)^{2}}{\nu} \int_{t_{0}}^{t_{N}}\left\|\pi_{t}(t)\right\|^{2} \mathrm{~d} \tau \\
& \left.+\frac{2 C}{\nu}(N \Delta t)\left[\boldsymbol{\xi}_{t}\right]_{\alpha}^{2}(\Delta t)^{2 \alpha}+\nu C(\Delta t)^{2} \int_{t_{0}}^{t_{N}}\left\|\nabla \boldsymbol{\xi}_{t}(\tau)\right\|^{2} \mathrm{~d} \tau+\left\|\mathbf{e}_{h}^{0}\right\|^{2}\right) .
\end{aligned}
$$

Applying the triangle inequality, we have

$$
\begin{gathered}
\left\|\boldsymbol{\xi}\left(t_{N}\right)-\boldsymbol{\xi}_{h}^{N}\right\|^{2}+\nu \Delta t \sum_{n=0}^{N-1}\left\|\nabla\left(\boldsymbol{\xi}^{n+\frac{1}{2}}-\boldsymbol{\xi}_{h}^{n+\frac{1}{2}}\right)\right\|^{2} \\
\leq 2\left\|\mathbf{e}_{h}^{N}\right\|^{2}+2 \nu \Delta t \sum_{n=0}^{N-1}\left\|\nabla \mathbf{e}_{h}^{n+\frac{1}{2}}\right\|^{2}+2\left\|\mathbf{e}_{I}^{N}\right\|^{2}+2 \nu \Delta t \sum_{n=0}^{N-1}\left\|\nabla \mathbf{e}_{I}^{n+\frac{1}{2}}\right\|^{2} .
\end{gathered}
$$

In conclusion, we have Theorem 4.2.

\section{Numerical experiments}

In this section, we give experiments of (1.1) with truncated time-space white noise (cylindrical Wiener process) and regularized time-space white noise.

\subsection{Numerical scheme}

With appropriately selected regularization parameter $\theta$, the stochastic forcing term can be guaranteed $\Delta_{\theta} \dot{\mathbf{W}}(t) \in L_{\mathbb{P}}^{2}\left(\Omega, \mathbf{H}^{-1}(D)\right)$. For a given sample $\omega \in \Omega, N_{n} \in \mathbb{N}$, suppose that the initial values $\boldsymbol{\eta}_{h}^{0}(\omega), \boldsymbol{\xi}_{h}^{0}(\omega) \in \mathbf{X}_{h}$ and $\zeta_{h}^{0}(\omega), \pi_{h}^{0}(\omega) \in Q_{h}$, for the stochastic Stokes equations, solve for $\left(\boldsymbol{\eta}_{h}^{n}(\omega), \zeta_{h}^{n}(\omega)\right) \in \mathbf{X}_{h} \times Q_{h}, n=1,2, \cdots, N$, such that for all $\left(\mathbf{v}_{h}, q_{h}\right) \in \mathbf{X}_{h} \times Q_{h}$ from

$$
\begin{aligned}
& \left(\frac{\boldsymbol{\eta}_{h}^{n+1}-\boldsymbol{\eta}_{h}^{n}}{\Delta t}, \mathbf{v}_{h}\right)+a\left(\boldsymbol{\eta}_{h}^{n+\frac{1}{2}}, \mathbf{v}_{h}\right)+b\left(\mathbf{v}_{h}, \zeta_{h}^{n+1}\right) \\
& =\left(\mathbf{f}_{1}^{n+\frac{1}{2}}, \mathbf{v}_{h}\right)+\frac{1}{\Delta t} \sum_{l=1}^{N_{h}} \lambda_{l}^{-\theta} \sigma\left(t_{n}\right)\left(\psi_{l}(x)\left(\boldsymbol{\beta}_{l}^{n+1}(\omega)-\boldsymbol{\beta}_{l}^{n}(\omega)\right), \mathbf{v}_{h}\right), \\
& b\left(\boldsymbol{\eta}_{h}^{n+1}, q_{h}\right)=0,
\end{aligned}
$$


and for the stochastic modified Navier-Stokes equations, solve for $\left(\boldsymbol{\xi}_{h}^{n}(\omega), \pi_{h}^{n}(\omega)\right) \in$ $\mathbf{X}_{h} \times Q_{h}$ from

$$
\begin{aligned}
& \left(\frac{\boldsymbol{\xi}_{h}^{n+1}-\boldsymbol{\xi}_{h}^{n}}{\Delta t}, \mathbf{v}_{h}\right)+a\left(\boldsymbol{\xi}_{h}^{n+\frac{1}{2}}, \mathbf{v}_{h}\right)+\tilde{c}\left(\boldsymbol{\xi}_{h}^{n}+\boldsymbol{\eta}_{h}^{n} ; \boldsymbol{\xi}_{h}^{n+\frac{1}{2}}+\boldsymbol{\eta}_{h}^{n+\frac{1}{2}}, \mathbf{v}_{h}\right) \\
& \quad+b\left(\mathbf{v}_{h}, \pi_{h}^{n+1}\right)=\left(\mathbf{f}^{n+\frac{1}{2}}-\mathbf{f}_{1}^{n+\frac{1}{2}}, \mathbf{v}_{h}\right), \\
& b\left(\boldsymbol{\xi}_{h}^{n+1}, q_{h}\right)=0 .
\end{aligned}
$$

\subsection{Deterministic case}

To demonstrate the effectiveness of our schemes, we first choose the deterministic Navier-Stokes equations as follows:

$$
\left\{\begin{array}{l}
\mathbf{u}_{t}-\nu \Delta \mathbf{u}+\mathbf{u} \cdot \nabla \mathbf{u}+\nabla p=\mathbf{f} \\
\nabla \cdot \mathbf{u}=0
\end{array}\right.
$$

on $D=[0,1] \times[0,1]$ and in the time interval $[0,1]$. Initial value $\mathbf{u}_{0}$ and the external force $\mathbf{f}$ are determined by the exact solutions

$$
\begin{gathered}
u_{1}:(x, y, t) \quad \mapsto 10 x^{2}(1-x)^{2}\left(y-3 y^{2}+2 y^{3}\right) \cos (2 \pi t), \\
u_{2}:(x, y, t) \mapsto-10 y^{2}(1-y)^{2}\left(x-3 x^{2}+2 x^{3}\right) \cos (2 \pi t), \\
p \quad:(x, y, t)
\end{gathered}
$$

To test our scheme, the original Navier-Stokes equations (5.1) are split into the Stokes equations (5.2) and the modified Navier-Stokes equations. For the Stokes equations,

$$
\left\{\begin{array}{l}
\boldsymbol{\eta}_{t}-\nu \Delta \boldsymbol{\eta}+\nabla \zeta=\mathbf{f}_{1}, \\
\nabla \cdot \boldsymbol{\eta}=0
\end{array}\right.
$$

Let the exact solutions $\boldsymbol{\eta}=\left(\eta_{1}, \eta_{2}\right), \zeta$ be half of $\mathbf{u}, p$ as follows,

$$
\begin{aligned}
\eta_{1}:(x, y, t) & \mapsto 5 x^{2}(1-x)^{2}\left(y-3 y^{2}+2 y^{3}\right) \cos (2 \pi t), \\
\eta_{2}:(x, y, t) & \mapsto-5 y^{2}(1-y)^{2}\left(x-3 x^{2}+2 x^{3}\right) \cos (2 \pi t), \\
\zeta:(x, y, t) & \mapsto 5(2 x-1)(2 y-1) \cos (2 \pi t) .
\end{aligned}
$$

The initial value $\boldsymbol{\eta}_{0}$ and the external force $\mathbf{f}_{1}$ are correspondingly determined. Then the modified Navier-Stokes equations with velocity field $\boldsymbol{\xi}=\left(\xi_{1}, \xi_{2}\right)$, pressure $\pi$ can be directly obtained by subtracting (5.2) from (5.1). The uniform triangulations $\left\{T_{h}\right\}_{h>0}$ are constructed by partitioning the unit square $D$ into $n \times n$ subrectangles and dividing each sub-square element into two triangles by the diagonal line. The mesh size of $T_{h}$ is denoted by $h=1 / n$. The finite element spaces are approximated by the stable $P_{2} / P_{1}$ Taylor-Hood elements for velocity and pressure, respectively.

In this experiment and subsequent experiments, we set the viscosity parameter $\nu=0.01$. To verify convergence in space, we fix a small $\Delta t=0.001$ and choose 
Table 1: Errors and convergence rates for the deterministic case.

\begin{tabular}{||c|cccccc||}
\hline \multirow{2}{*}{$\Delta x$} & \multicolumn{2}{|c}{ Stokes } & \multicolumn{2}{c||}{ Modified Navier-Stokes } & \multicolumn{2}{c||}{ Navier-Stokes } \\
\cline { 2 - 7 } & $e\left(\eta_{1}\right)$ & $e\left(\eta_{2}\right)$ & $e\left(\xi_{1}\right)$ & $e\left(\xi_{2}\right)$ & $e\left(u_{1}\right)$ & $e\left(u_{2}\right)$ \\
\hline $1 / 8$ & $7.123 \mathrm{E}-05$ & $7.123 \mathrm{E}-05$ & $7.143 \mathrm{E}-05$ & $7.117 \mathrm{E}-05$ & $1.426 \mathrm{E}-04$ & $1.424 \mathrm{E}-04$ \\
\hline $1 / 16$ & $9.235 \mathrm{E}-06$ & $9.235 \mathrm{E}-06$ & $9.242 \mathrm{E}-06$ & $9.233 \mathrm{E}-06$ & $1.847 \mathrm{E}-05$ & $1.847 \mathrm{E}-05$ \\
\hline $1 / 32$ & $1.167 \mathrm{E}-06$ & $1.167 \mathrm{E}-06$ & $1.167 \mathrm{E}-06$ & $1.167 \mathrm{E}-06$ & $2.333 \mathrm{E}-06$ & $2.333 \mathrm{E}-06$ \\
\hline $1 / 64$ & $1.451 \mathrm{E}-07$ & $1.451 \mathrm{E}-07$ & $1.451 \mathrm{E}-07$ & $1.451 \mathrm{E}-07$ & $2.903 \mathrm{E}-07$ & $2.903 \mathrm{E}-07$ \\
\hline $\mathcal{O}\left(\Delta x^{\beta}\right)$ & 2.980 & 2.980 & 2.981 & 2.980 & 2.981 & 2.980 \\
\hline \hline \multirow{2}{*}{$\Delta t$} & \multicolumn{2}{|c}{ Stokes } & \multicolumn{2}{c}{ Modified Navier-Stokes } & \multicolumn{2}{c||}{ Navier-Stokes } \\
\cline { 2 - 8 } & $e\left(\eta_{1}\right)$ & $e\left(\eta_{2}\right)$ & $e\left(\xi_{1}\right)$ & $e\left(\xi_{2}\right)$ & $e\left(u_{1}\right)$ & $e\left(u_{2}\right)$ \\
\hline $1 / 60$ & $5.152 \mathrm{E}-06$ & $5.152 \mathrm{E}-06$ & $5.475 \mathrm{E}-06$ & $5.475 \mathrm{E}-06$ & $1.047 \mathrm{E}-05$ & $1.047 \mathrm{E}-05$ \\
\hline $1 / 80$ & $2.896 \mathrm{E}-06$ & $2.896 \mathrm{E}-06$ & $3.170 \mathrm{E}-06$ & $3.170 \mathrm{E}-06$ & $5.934 \mathrm{E}-06$ & $5.934 \mathrm{E}-06$ \\
\hline $1 / 100$ & $1.852 \mathrm{E}-06$ & $1.852 \mathrm{E}-06$ & $2.095 \mathrm{E}-06$ & $2.095 \mathrm{E}-06$ & $3.832 \mathrm{E}-06$ & $3.832 \mathrm{E}-06$ \\
\hline $1 / 120$ & $1.285 \mathrm{E}-06$ & $1.285 \mathrm{E}-06$ & $1.506 \mathrm{E}-06$ & $1.506 \mathrm{E}-06$ & $2.688 \mathrm{E}-06$ & $2.688 \mathrm{E}-06$ \\
\hline $\mathcal{O}\left(\Delta t^{r}\right)$ & 2.003 & 2.003 & 1.864 & 1.864 & 1.962 & 1.962 \\
\hline
\end{tabular}

a sequence of $\Delta h_{i}=1 / 2^{i}, i=3, \cdots, 7$. The numerical solutions on a fine mesh with $\Delta h=1 / 128$, are regarded as the true solutions for the original Navier-Stokes equations, the Stokes equations and the modified Navier-Stokes equations. To verify the convergence rate in time, we choose a fixed small $\Delta h=1 / 64$ and a sequence of $\Delta t_{i} \in\{1 / 60,1 / 80,1 / 100,1 / 120\}$, then the true solutions of (5.1) and (5.2) are approximated by the numerical solutions computed with a small time step $\Delta t=1 / 1200$. We use the notation $e(\cdot)$ to represent the $L^{2}(D)$ error at the final time instant $t=T$. Table 1 tells us that the convergence rates for velocities $\boldsymbol{\eta}(T), \boldsymbol{\xi}(T), \mathbf{u}(T)$ are all 3 in space and 2 in time which are consistent with the classical finite element theoretical results.

\subsection{Truncated white noise}

Adding the stochastic noise to the deterministic Navier-Stokes equations (5.1) leads to the stochastic Navier-Stokes equations. The space-time white noise actually has very poor regularity. As it is already pointed out in Corollary 3.1, for a linear parabolic equation with space-time white noise in $2 D$, the solution will not belong to $L_{\mathbb{P}}^{2}\left(\Omega, L^{2}(D)\right)$. In this part, we consider the properties of (1.1) with truncated space-time white noise numerically. The space-time white noise is characterized by a cylindrical Wiener process. We extract a subset of $\left\{\psi_{l}\right\}_{1}^{N_{h}}$ of $L^{2}(D)$ from $-\Delta \psi_{l}=\lambda_{l} \psi_{l}$ on domain $[0,1] \times[0,1]$ with homogeneous boundary condition. Thus, the truncated space-time white noise is defined as

$$
\mathrm{d} \mathbf{W}_{h}(t)=\frac{2}{N_{h}} \sum_{i=1}^{N_{h}} \sum_{j=1}^{N_{h}} \sin (i \pi x) \sin (j \pi y) \mathrm{d} \boldsymbol{\beta}_{i, j}(t),
$$

where $\left\{\boldsymbol{\beta}_{i, j}(t)=\left(\beta_{i}, \beta_{j}\right)\right\}$ are the mutually independent one dimensional standard Brownian motions. In this test, we set the total truncated number $128 \times 128=16384$ 
Table 2: Errors and convergence rates in space with truncated white noise.

\begin{tabular}{||c|cccccc||}
\hline \multirow{2}{*}{$\Delta x$} & \multicolumn{2}{|c}{ Stokes } & \multicolumn{2}{c||}{ Modified Navier-Stokes } & \multicolumn{2}{c||}{ Navier-Stokes } \\
\cline { 2 - 7 } & $e_{s}\left(\eta_{1}\right)$ & $e_{s}\left(\eta_{2}\right)$ & $e_{s}\left(\xi_{1}\right)$ & $e_{s}\left(\xi_{2}\right)$ & $e_{s}\left(u_{1}\right)$ & $e_{s}\left(u_{2}\right)$ \\
\hline $1 / 8$ & $9.004 \mathrm{E}-02$ & $9.054 \mathrm{E}-02$ & $9.518 \mathrm{E}-03$ & $9.617 \mathrm{E}-03$ & $8.998 \mathrm{E}-02$ & $9.045 \mathrm{E}-02$ \\
\hline $1 / 16$ & $5.469 \mathrm{E}-02$ & $5.498 \mathrm{E}-02$ & $3.165 \mathrm{E}-03$ & $3.197 \mathrm{E}-03$ & $5.467 \mathrm{E}-02$ & $5.496 \mathrm{E}-02$ \\
\hline $1 / 32$ & $2.724 \mathrm{E}-02$ & $2.728 \mathrm{E}-02$ & $9.309 \mathrm{E}-04$ & $9.320 \mathrm{E}-04$ & $2.725 \mathrm{E}-02$ & $2.727 \mathrm{E}-02$ \\
\hline $1 / 64$ & $1.214 \mathrm{E}-02$ & $1.215 \mathrm{E}-02$ & $1.831 \mathrm{E}-04$ & $1.821 \mathrm{E}-04$ & $1.214 \mathrm{E}-02$ & $1.215 \mathrm{E}-02$ \\
\hline $\mathcal{O}\left(\Delta x^{\beta}\right)$ & 0.968 & 0.970 & 1.887 & 1.895 & 0.967 & 0.970 \\
\hline \hline \multirow{2}{*}{$\Delta x$} & \multicolumn{2}{|c}{ Stokes } & \multicolumn{1}{c}{ Modified Navier-Stokes } & \multicolumn{2}{c||}{ Navier-Stokes } \\
\cline { 2 - 7 } & $e_{w}\left(\eta_{1}\right)$ & $e_{w}\left(\eta_{2}\right)$ & $e_{w}\left(\xi_{1}\right)$ & $e_{w}\left(\xi_{2}\right)$ & $e_{w}\left(u_{1}\right)$ & $e_{w}\left(u_{2}\right)$ \\
\hline $1 / 8$ & $6.116 \mathrm{E}-03$ & $6.616 \mathrm{E}-03$ & $8.171 \mathrm{E}-04$ & $9.252 \mathrm{E}-04$ & $6.207 \mathrm{E}-03$ & $6.498 \mathrm{E}-03$ \\
\hline $1 / 16$ & $3.773 \mathrm{E}-03$ & $3.970 \mathrm{E}-03$ & $2.434 \mathrm{E}-04$ & $2.596 \mathrm{E}-04$ & $3.790 \mathrm{E}-03$ & $3.998 \mathrm{E}-03$ \\
\hline $1 / 32$ & $1.860 \mathrm{E}-03$ & $1.899 \mathrm{E}-03$ & $6.862 \mathrm{E}-05$ & $5.982 \mathrm{E}-05$ & $1.864 \mathrm{E}-03$ & $1.898 \mathrm{E}-03$ \\
\hline $1 / 64$ & $8.681 \mathrm{E}-04$ & $8.569 \mathrm{E}-04$ & $1.230 \mathrm{E}-05$ & $1.254 \mathrm{E}-05$ & $8.679 \mathrm{E}-04$ & $8.571 \mathrm{E}-04$ \\
\hline $\mathcal{O}\left(\Delta x^{\beta}\right)$ & 0.947 & 0.991 & 1.999 & 2.073 & 0.954 & 0.984 \\
\hline
\end{tabular}

with $N_{h}=128, \sigma(t, x)=1$ and $\Delta_{\theta}=I$ with $\theta=0$. Then the strong and weak $L_{\mathbb{P}}^{2}$ errors at the final time instant $t=T$ are defined as

$$
e_{s}(\mathbf{u})=\left(\mathbb{E}\left[\left\|\mathbf{u}_{h}^{N}-\mathbf{u}\left(t_{N}\right)\right\|^{2}\right]\right)^{1 / 2}, \quad e_{w}(\mathbf{u})=\left\|\mathbb{E}\left[\mathbf{u}_{h}^{N}\right]-\mathbb{E}\left[\mathbf{u}\left(t_{N}\right)\right]\right\|
$$

respectively.

In our tests, we set $M=200$ the number of the realizations. Table 2 shows that the convergence rates for the stochastic modified Navier-Stokes equations are higher than the stochastic Stokes equations. The reason is that the stochastic modified NavierStokes equations are associated with a better regularized noise than the stochastic Stokes equations. Therefore, it is meaningful to do pathwise theoretical analyses. Table 2 and Table 3 show that the weak convergence errors are always smaller than the strong convergence error. In view of the Monte Carlo error, the more number of the simulations, the better results for the weak convergence test. Table 3 shows that the regularity in time direction is somehow poor for the stochastic Stokes equation with truncated white noise. While, the stochastic modified Navier-Stokes equations have a improved regularities. It shows that the Martingale regularization method is effective to simulate the stochastic problems.

\subsection{Truncated regularized white noise}

Let the regularization parameter $\theta$ be a real positive number, the noise term $\Delta_{\theta} \mathrm{dW}$ will have a better regularity than non-regularized $\mathrm{dW}$. In turn, the solution of (1.1) will be much smoother. As the truncated case in 5.3, the regularized noise is written as

$$
(-\Delta)^{-\theta} \mathrm{d} \mathbf{W}_{h}=\sum_{i=1}^{N_{h}} \sum_{j=1}^{N_{h}} \frac{2}{N_{h}}\left(\pi^{2} i^{2}+\pi^{2} j^{2}\right)^{-\theta} \sin (i \pi x) \sin (j \pi y) \mathrm{d} \boldsymbol{\beta}_{i, j} .
$$


Table 3: Errors and convergence rates in time with truncated white noise.

\begin{tabular}{||c|cccccc||}
\hline \multirow{2}{*}{$\Delta t$} & \multicolumn{2}{|c}{ Stokes } & \multicolumn{2}{c||}{ Modified Navier-Stokes } & \multicolumn{2}{c||}{ Navier-Stokes } \\
\cline { 2 - 7 } & $e_{s}\left(\eta_{1}\right)$ & $e_{s}\left(\eta_{2}\right)$ & $e_{s}\left(\xi_{1}\right)$ & $e_{s}\left(\xi_{2}\right)$ & $e_{s}\left(u_{1}\right)$ & $e_{s}\left(u_{2}\right)$ \\
\hline $1 / 60$ & $2.176 \mathrm{E}-02$ & $2.175 \mathrm{E}-02$ & $2.648 \mathrm{E}-04$ & $2.648 \mathrm{E}-04$ & $2.176 \mathrm{E}-02$ & $2.175 \mathrm{E}-02$ \\
\hline $1 / 80$ & $2.030 \mathrm{E}-02$ & $2.028 \mathrm{E}-02$ & $2.281 \mathrm{E}-04$ & $2.285 \mathrm{E}-04$ & $2.030 \mathrm{E}-02$ & $2.028 \mathrm{E}-02$ \\
\hline $1 / 100$ & $1.908 \mathrm{E}-02$ & $1.909 \mathrm{E}-02$ & $2.024 \mathrm{E}-04$ & $2.026 \mathrm{E}-04$ & $1.908 \mathrm{E}-02$ & $1.909 \mathrm{E}-02$ \\
\hline $1 / 120$ & $1.806 \mathrm{E}-02$ & $1.807 \mathrm{E}-02$ & $1.832 \mathrm{E}-04$ & $1.833 \mathrm{E}-04$ & $1.806 \mathrm{E}-02$ & $1.807 \mathrm{E}-02$ \\
\hline $\mathcal{O}\left(\Delta t^{r}\right)$ & 0.268 & 0.267 & 0.531 & 0.531 & 0.268 & 0.267 \\
\hline \hline \multirow{2}{*}{$\Delta t$} & \multicolumn{2}{|c}{ Stokes } & \multicolumn{2}{c}{ Modified Navier-Stokes } & \multicolumn{2}{c||}{ Navier-Stokes } \\
\cline { 2 - 7 } & $e_{w}\left(\eta_{1}\right)$ & $e_{w}\left(\eta_{2}\right)$ & $e_{w}\left(\xi_{1}\right)$ & $e_{w}\left(\xi_{2}\right)$ & $e_{w}\left(u_{1}\right)$ & $e_{w}\left(u_{2}\right)$ \\
\hline $1 / 60$ & $1.536 \mathrm{E}-03$ & $1.543 \mathrm{E}-03$ & $1.967 \mathrm{E}-05$ & $1.904 \mathrm{E}-05$ & $1.536 \mathrm{E}-03$ & $1.543 \mathrm{E}-03$ \\
\hline $1 / 80$ & $1.448 \mathrm{E}-03$ & $1.427 \mathrm{E}-03$ & $1.644 \mathrm{E}-05$ & $1.667 \mathrm{E}-05$ & $1.448 \mathrm{E}-03$ & $1.427 \mathrm{E}-03$ \\
\hline $1 / 100$ & $1.336 \mathrm{E}-03$ & $1.352 \mathrm{E}-03$ & $1.467 \mathrm{E}-05$ & $1.443 \mathrm{E}-05$ & $1.336 \mathrm{E}-03$ & $1.352 \mathrm{E}-03$ \\
\hline $1 / 120$ & $1.280 \mathrm{E}-03$ & $1.272 \mathrm{E}-03$ & $1.352 \mathrm{E}-05$ & $1.295 \mathrm{E}-05$ & $1.280 \mathrm{E}-03$ & $1.272 \mathrm{E}-03$ \\
\hline $\mathcal{O}\left(\Delta t^{r}\right)$ & 0.271 & 0.275 & 0.542 & 0.562 & 0.271 & 0.275 \\
\hline
\end{tabular}

Table 4: Errors and convergence rates in space with regularized truncated white noise.

\begin{tabular}{||c|cccccc||}
\hline \multirow{2}{*}{$\Delta x$} & \multicolumn{2}{|c}{ Stokes } & \multicolumn{2}{c||}{ Modified Navier-Stokes } & \multicolumn{2}{c||}{ Navier-Stokes } \\
\cline { 2 - 7 } & $e_{s}\left(\eta_{1}\right)$ & $e_{s}\left(\eta_{2}\right)$ & $e_{s}\left(\xi_{1}\right)$ & $e_{s}\left(\xi_{2}\right)$ & $e_{s}\left(u_{1}\right)$ & $e_{s}\left(u_{2}\right)$ \\
\hline $1 / 8$ & $2.397 \mathrm{E}-04$ & $2.392 \mathrm{E}-04$ & $7.204 \mathrm{E}-05$ & $7.176 \mathrm{E}-05$ & $2.699 \mathrm{E}-04$ & $2.691 \mathrm{E}-04$ \\
\hline $1 / 16$ & $1.036 \mathrm{E}-04$ & $1.039 \mathrm{E}-04$ & $9.622 \mathrm{E}-06$ & $9.609 \mathrm{E}-06$ & $1.048 \mathrm{E}-04$ & $1.051 \mathrm{E}-04$ \\
\hline $1 / 32$ & $4.217 \mathrm{E}-05$ & $4.211 \mathrm{E}-05$ & $1.336 \mathrm{E}-06$ & $1.337 \mathrm{E}-06$ & $4.222 \mathrm{E}-05$ & $4.216 \mathrm{E}-05$ \\
\hline $1 / 64$ & $1.539 \mathrm{E}-05$ & $1.540 \mathrm{E}-05$ & $1.801 \mathrm{E}-07$ & $1.802 \mathrm{E}-07$ & $1.539 \mathrm{E}-05$ & $1.540 \mathrm{E}-05$ \\
\hline $\mathcal{O}\left(\Delta x^{\beta}\right)$ & 1.318 & 1.317 & 2.878 & 2.876 & 1.371 & 1.370 \\
\hline \hline \multirow{2}{*}{$\Delta x$} & \multicolumn{2}{|c}{ Stokes } & Modified Navier-Stokes & Navier-Stokes \\
\cline { 2 - 7 } & $e_{w}\left(\eta_{1}\right)$ & $e_{w}\left(\eta_{2}\right)$ & $e_{w}\left(\xi_{1}\right)$ & $e_{w}\left(\xi_{2}\right)$ & $e_{w}\left(u_{1}\right)$ & $e_{w}\left(u_{2}\right)$ \\
\hline $1 / 8$ & $7.312 \mathrm{E}-05$ & $7.310 \mathrm{E}-05$ & $7.145 \mathrm{E}-05$ & $7.118 \mathrm{E}-05$ & $1.437 \mathrm{E}-04$ & $1.434 \mathrm{E}-04$ \\
\hline $1 / 16$ & $1.167 \mathrm{E}-05$ & $1.168 \mathrm{E}-05$ & $9.247 \mathrm{E}-06$ & $9.235 \mathrm{E}-06$ & $1.989 \mathrm{E}-05$ & $1.984 \mathrm{E}-05$ \\
\hline $1 / 32$ & $3.243 \mathrm{E}-06$ & $3.181 \mathrm{E}-06$ & $1.168 \mathrm{E}-06$ & $1.167 \mathrm{E}-06$ & $3.823 \mathrm{E}-06$ & $3.774 \mathrm{E}-06$ \\
\hline $1 / 64$ & $1.117 \mathrm{E}-06$ & $1.102 \mathrm{E}-06$ & $1.454 \mathrm{E}-07$ & $1.453 \mathrm{E}-07$ & $1.145 \mathrm{E}-06$ & $1.130 \mathrm{E}-06$ \\
\hline $\mathcal{O}\left(\Delta x^{\beta}\right)$ & 1.995 & 2.003 & 2.981 & 2.979 & 2.329 & 2.336 \\
\hline
\end{tabular}

With increasing the parameter $\theta$, the noise term will become more and more regularity. In this test, we set the $\theta=0.6, \sigma(t)=1$. After regularizing the noise in space, it is nontrivial to verify that $(-\Delta)^{-0.6} \dot{\mathbf{W}} \in \mathbf{L}_{\mathbb{P}}^{2}\left(\Omega, \mathbf{H}^{-1}(D)\right)$. In this case, the pathwise modified Navier-Stokes equations can be considered to be a classical deterministic case in space. Tables 4, 5 illustrate that strong and weak convergence results for the stochastic Stokes equations become much better than the cases of the truncated white noise in Tables 2 and 3. For the modified Navier-Stokes equations, in Table 4, the convergence rates are almost 3 which means that the modified Navier-Stokes equations have better regularity than the stochastic Stokes equations. The weak convergence rates are always higher than the strong convergence rates. Compared with Table 3, Table 5 shows better convergence results in time. The computational results are consistent with the 
Table 5: Errors and convergence rates in time with regularized truncated white noise.

\begin{tabular}{||c|cccccc||}
\hline \multirow{2}{*}{$\Delta t$} & \multicolumn{2}{|c}{ Stokes } & \multicolumn{2}{c|}{ Modified Navier-Stokes } & \multicolumn{2}{c||}{ Navier-Stokes } \\
\cline { 2 - 7 } & $e_{s}\left(\eta_{1}\right)$ & $e_{s}\left(\eta_{2}\right)$ & $e_{s}\left(\xi_{1}\right)$ & $e_{s}\left(\xi_{2}\right)$ & $e_{s}\left(u_{1}\right)$ & $e_{s}\left(u_{2}\right)$ \\
\hline $1 / 60$ & $4.232 \mathrm{E}-05$ & $4.232 \mathrm{E}-05$ & $5.778 \mathrm{E}-06$ & $5.782 \mathrm{E}-06$ & $4.333 \mathrm{E}-05$ & $4.334 \mathrm{E}-05$ \\
\hline $1 / 80$ & $3.594 \mathrm{E}-05$ & $3.593 \mathrm{E}-05$ & $3.455 \mathrm{E}-06$ & $3.461 \mathrm{E}-06$ & $3.633 \mathrm{E}-05$ & $3.633 \mathrm{E}-05$ \\
\hline $1 / 100$ & $3.164 \mathrm{E}-05$ & $3.165 \mathrm{E}-05$ & $2.367 \mathrm{E}-06$ & $2.373 \mathrm{E}-06$ & $3.184 \mathrm{E}-05$ & $3.185 \mathrm{E}-05$ \\
\hline $1 / 120$ & $2.848 \mathrm{E}-05$ & $2.850 \mathrm{E}-05$ & $1.760 \mathrm{E}-06$ & $1.764 \mathrm{E}-06$ & $2.860 \mathrm{E}-05$ & $2.862 \mathrm{E}-05$ \\
\hline$O\left(\Delta t^{r}\right)$ & 0.571 & 0.570 & 1.717 & 1.714 & 0.599 & 0.599 \\
\hline \hline \multirow{2}{*}{$\Delta t$} & \multicolumn{2}{|c}{ Stokes } & \multicolumn{2}{c}{ Modified Navier-Stokes } & Navier-Stokes \\
\cline { 2 - 7 } & $e_{w}\left(\eta_{1}\right)$ & $e_{w}\left(\eta_{2}\right)$ & $e_{w}\left(\xi_{1}\right)$ & $e_{w}\left(\xi_{2}\right)$ & $e_{w}\left(u_{1}\right)$ & $e_{w}\left(u_{2}\right)$ \\
\hline $1 / 60$ & $5.896 \mathrm{E}-06$ & $5.912 \mathrm{E}-06$ & $5.465 \mathrm{E}-06$ & $5.472 \mathrm{E}-06$ & $1.081 \mathrm{E}-05$ & $1.086 \mathrm{E}-05$ \\
\hline $1 / 80$ & $3.758 \mathrm{E}-06$ & $3.786 \mathrm{E}-06$ & $3.158 \mathrm{E}-06$ & $3.160 \mathrm{E}-06$ & $6.339 \mathrm{E}-06$ & $6.390 \mathrm{E}-06$ \\
\hline $1 / 100$ & $2.880 \mathrm{E}-06$ & $2.924 \mathrm{E}-06$ & $2.081 \mathrm{E}-06$ & $2.090 \mathrm{E}-06$ & $4.409 \mathrm{E}-06$ & $4.450 \mathrm{E}-06$ \\
\hline $1 / 120$ & $2.390 \mathrm{E}-06$ & $2.393 \mathrm{E}-06$ & $1.495 \mathrm{E}-06$ & $1.501 \mathrm{E}-06$ & $3.350 \mathrm{E}-06$ & $3.370 \mathrm{E}-06$ \\
\hline$O\left(\Delta t^{r}\right)$ & 1.305 & 1.302 & 1.872 & 1.867 & 1.692 & 1.689 \\
\hline
\end{tabular}

traditional acknowledge of stochastic analysis. Martingale regularization method with noise regularization feasibly improves computational efficiency of the system (1.1).

Acknowledgements This publication was supported in part by the US Air Force Office of Scientific Research grant FA9550-15-1-0001.

\section{Conclusions}

In this paper, we studied the stochastic Navier-Stokes equations with a Martingale regularization method. The noise is extended to a larger non-smooth class with a space regularizer $\Delta_{\theta}$. Under sufficient assumption, we presented the pathwise convergence analysis for the modified Navier-Stokes equations. We compared the stochastic NavierStokes equations with truncated/regularized time-space white noise numerically. The computational results provide an evidence that this kind of regularization method is powerful, effective and efficient. Beyond the stochastic Navier-Stokes system, Martingale regularization method is meaningful to other nonlinear SPDEs.

\section{References}

[1] S. ChaI, Y. CAO, Y. ZoU AND W. ZHAO, Conforming finite element methods for the stochastic Cahn-Hilliard-Cook equation, Appl. Numer. Math., 124 (2018), pp. 44-56.

[2] G. Da Prato and A. Debussche, Two-dimensional Navier-Stokes equations driven by a space-time white noise, J. Funct. Anal., 196 (2002), pp. 180-210.

[3] G. DA PRATO AND J. ZABCZYK, Stochastic Equations in Infinite Dimensions, vol. 44 of Encyclopedia of Mathematics and Its Applications, Cambridge University Press, Cambridge, 1992. 
[4] L. Du AND T. ZHANG, Global existence of axisymmetric pathwise solutions for stochastic three-dimensional axisymmetric Navier-Stokes equations, East. Asia. J. Appl. Math., 9 (2019), pp. 447-464.

[5] F. Flandoli AND D. GA̧TAREK, Martingale and stationary solutions for stochastic NavierStokes equations, Probab. Theory Related Fields, 102 (1995), pp. 367-391.

[6] M. GunZburger AND W. ZHAO, Descriptions, discretizations and comparisons of time/space colored and white noise forcings of the Navier-Stokes equations, SIAM J. Sci. Comput., 41(4) (2019), pp. A2579-A2602.

[7] M. D. GunZburger, L. Hou AND J. Ming, Stochastic steady-state Navier-Stokes equations with additive random noise, J. Sci. Comput., 66 (2016), pp. 672-691.

[8] L. GUO, A. NARAYAN AND T. ZHOU, Constructing least squares polynomial approximations, SIAM Rev., to appear, (2019).

[9] R. INGRAM, Unconditional convergence of high-order extrapolations of the Crank-Nicolson, finite element method for the Navier-Stokes equations, Int. J. Numer. Anal. Model., 10 (2013), pp. 257-297.

[10] G. J. LORD, C. E. Powell AND T. Shardlow, An Introduction to Computational Stochastic PDEs, Cambridge Texts in Applied Mathematics, Cambridge University Press, New York, 2014.

[11] J.-L. Menaldi And S. S. SRITHARAN, Stochastic 2-D Navier-Stokes equation, Appl. Math. Optim., 46 (2002), pp. 31-53.

[12] R. MikUlevicius, On the Cauchy problem for stochastic Stokes equations, SIAM J. Math. Anal., 34 (2002), pp. 121-141.

[13] R. Mikulevicius AND B. L. Rozovskir, Global $L_{2}$-solutions of stochastic Navier-Stokes equations, Ann. Probab., 33 (2005), pp. 137-176.

[14] A. NARAYAn AND T. ZHOU, Stochastic collocation on unstructured multivariate meshes, Commun. Comput. Phys., 18 (2015), pp. 1-36.

[15] T. TANG AND T. ZHOU, Recent developments in high order numerical methods for uncertainty quantification, Scientia Sinica Mathematica, 45 (2015), pp. 891-928.

[16] V. ThомÉE, Galerkin Finite Element Methods for Parabolic Problems, vol. 25 of Springer Series in Computational Mathematics, Springer-Verlag, Berlin, Second ed., 2006.

[17] W. ZHAO, Comparison of Different Noise Forcings, Regularization of Noise, and Optimal Control for the Stochastic Navier-Stokes Equation, ProQuest LLC, Ann Arbor, MI, 2017, Thesis (Ph.D.)-The Florida State University.

[18] H. ZHU, Y. Zou, S. CHAI AND C. ZHOU, Numerical approximation to a stochastic parabolic PDE with weak Galerkin method, Numer. Math. Theory Methods Appl., 11 (2018), pp. 604-617. 